\title{
Studienwahlmotive von Bewerber- innen und Bewerbern auf ein Lehramtsstudium und auf andere Studiengänge
}

\section{Studiengangübergreifende Vergleiche und Profilanalysen}

\author{
Robin Göller (1) und Michael Besser \\ Institut für Mathematik und ihre Didaktik, Leuphana Universität Lüneburg, Lüneburg, Deutschland
}

\begin{abstract}
Zusammenfassung: Studienwahlmotive von Lehramtsstudentinnen und -studenten sind in den vergangenen Jahren vermehrt in den Fokus von Forschungsvorhaben gerückt und erweisen sich als wichtiger Prädiktor für Studienerfolg und den Aufbau professioneller Kompetenzen. Dabei zeigt sich insbesondere das Erwartungs-Wert-Modell der Motivation als geeignete theoretische Grundlage zur Klassifikation und Operationalisierung verschiedener Studienwahlmotive. Zudem tragen die Betrachtung von Motivprofilen und Vergleiche verschiedener Lehramtsstudiengänge sowie verschiedener Länder zum besseren Verständnis von Studienwahlentscheidungen bei. Studiengangübergreifende Vergleiche, die über Lehramtsstudiengänge hinausgehen und Studienwahlmotive mittels reliabler Fragebogenskalen erfassen, sind jedoch kaum zu finden. Für den vorliegenden Beitrag wurde deshalb durch Adaption bestehender Instrumente ein Fragebogen zur studiengangübergreifenden Erhebung von Studienwahlmotiven entwickelt und von 690 Bewerberinnen und Bewerbern auf insgesamt 13 verschiedene Studiengänge bearbeitet. Es zeigt sich, dass sich die sechs Studienwahlmotive fachliche Fähigkeitsüberzeugung, fachliches Interesse, berufliches Interesse, gesellschaftlicher Wert des Studiums, persönliche Nützlichkeit und geringe Schwierigkeit des Studiums auch studiengangübergreifend reliabel (Cronbachs $\alpha>$.69) erfassen lassen und dass sich Bewerberinnen und Bewerber auf verschiedene Studiengänge signifikant in diesen Studienwahlmotiven unterscheiden ( $p \leq 0.003$ ). Insbesondere zeigt sich, dass das Studienwahlmotiv Fachinteresse bei Bewerberinnen und Bewerbern auf ein Lehramtsstudium niedriger als in anderen Studienganggruppen ausfällt (Cohens $d=-0.45$ ), und dass alle anderen erhobenen Studienwahlmotive bei Bewerberinnen und Bewerbern auf ein Lehramtsstudium am höchsten ausgeprägt sind - mit großen Effekten bei beruflichem Interesse $(d=1.39)$ und der persönlichen Nützlichkeit $(d=1.07)$ sowie mittleren Effekten in der fachlichen Fähigkeitsüberzeugung $(d=0.56)$ und dem gesellschaftlichen Wert des Studiums $(d=0.47)$. Eine personenzentrierte Betrachtung von Studienwahlmotiven über Motivprofile verdeutlicht darüber hinaus, dass für die Entscheidung für ein Lehramtsstudium vor allem das Zusammenspiel verschiedener (nicht nur inhaltlich fachlich begründeter) Studienwahlmotive relevant ist, wohingegen in einigen nicht lehramtsspezifischen Studiengängen insbesondere fachliche Interessen überwiegen. Die Ergebnisse werden mit Blick auf bisherige Forschungsergebnisse und ihre mögliche Relevanz für die praktische Ausgestaltung von universitären Lehrveranstaltungen diskutiert.
\end{abstract}

Schlüsselwörter: Studienwahlmotive, Studienwahl, Motivation, Lehramtsstudium, Hochschuldidaktik

Study choice motives of applicants for a teacher training programme and for other study programmes - Comparisons across study programmes and profile analyses

Abstract: In recent years, research projects have increasingly focused on the study choice motives of student teachers which have proven to be an important predictor of academic success and the development of professional competencies. In particular, the expectancy-value theory of motivation has proven to be a suitable theoretical basis for the classification and operationalisation of various motives for choosing a study programme. In addition, the examination of motivational profiles and comparisons of different teacher training programmes and different countries, contribute to a better understanding of study choices. However, comparisons that go beyond teacher training programmes and measure study choice motives with reliable questionnaire scales are hardly to be found. Therefore, for the present paper, a questionnaire for a cross-degree survey of study choice motives was developed by adapting existing instruments and completed by 690 applicants of a total of 13 different study programmes. Results show that the six motives for choosing a study programme - perceived subject specific ability, subjectspecific interest, professional interest, social value of the study programme, personal utility, and low difficulty of the study programme - can also be reliably (Cronbach's $\alpha>$.69) measured across study programmes and that applicants for different study programmes differ significantly in these motives $(p \leq 0.003)$. In particular, it was found that subject-specific interest as a study choice motive is lower among applicants for teacher training programmes than in other study programme groups (Cohen's $d=-0.45$ ), and that all other surveyed motives for choosing a study programme are highest among applicants for teacher training programmes - with large effects for professional interest $(d=1.39)$ and 
personal utility $(d=1.07)$, and medium effects for perceived subject-specific ability $(d=0.56)$ and the social value of the study programme $(d=$ 0.47). A person-centred analysis of study choice motives via motive profiles also illustrates that the interplay of different (not only subject-related) study choice motives is particularly relevant to the decision to study for a teaching profession, whereas in some programmes that are not specific to teaching professions, particular subject interests predominate. The results are discussed with regard to previous research results and their possible relevance for the practical design of university courses.

Keywords: Study choice motives, study choice, motivation, teacher training, higher education

\section{Einleitung}

Die Motivation, mit der sich angehende Studentinnen und Studenten für ein Lehramtsstudium entscheiden, ist ein wichtiger Prädiktor für Studienerfolg und den Aufbau professioneller Kompetenzen: Insbesondere für intrinsische Studienwahlmotive finden sich statistisch bedeutsame $\mathrm{Zu}$ sammenhänge zu Studienstrategienutzung, Studienzufriedenheit (Künsting \& Lipowsky, 2011; Wach et al., 2016), höherem Karriereoptimismus und geringer ausgeprägten Bournout-Symptomen (McLean et al., 2019), Lernzielorientierungen, pädagogischem Wissen (König et al., 2018), zu Unterrichtsqualität von Lehramtsstudentinnen und Lehramtsstudenten in Praktika (Biermann et al., 2019; Hanfstingl \& Mayr, 2007) sowie zu Unterrichtsqualität und beruflicher Zufriedenheit von Lehrerinnen und Lehrern (Hanfstingl \& Mayr, 2007). Die Frage, aufgrund welcher Motivation sich Personen für ein Lehramtsstudium entscheiden, ist aber nicht nur von individueller Bedeutung für die betreffenden Personen und deren Erfolgschancen in Studium und Beruf, sondern auch von gesellschaftlicher Relevanz, da Lehrerinnen und Lehrer einen nicht zu unterschätzenden Beitrag für die Entwicklung und Ausbildung der nachfolgenden Generation leisten (Baumert \& Kunter, 2006; Kunter \& Pohlmann, 2009; Richardson \& Watt, 2006). Ausgehend von dieser Bedeutung von Studienwahlmotiven sowohl für den individuellen Erfolg als auch das gesellschaftliche Wohl stellt sich ganz grundsätzlich die Frage, welche Personen sich aus welchen Gründen für ein Lehramtsstudium und damit den Lehrerinnen- und Lehrerberuf entscheiden (Fray \& Gore, 2018; Richardson \& Watt, 2006; Roloff Henoch et al., 2015).

Dementsprechend sind Studienwahlmotive von Lehramtsstudentinnen und -studenten sowohl mit Blick auf die Identifikation typischer Motivationen zur Aufnahme eines Lehramtsstudiums als auch hinsichtlich ihrer Bedeutung für Studienerfolg und den Aufbau professioneller Kompetenzen in den vergangenen Jahren national und international vielfach untersucht worden (z. B. Biermann et al., 2019; Billich-Knapp et al., 2012; Dörrenbächer-Ulrich et al., 2019; Fray \& Gore, 2018; Goller et al., 2019; König et al., 2018; Künsting \& Lipowsky, 2011; Pohlmann \& Möller, 2010; Rutsch et al., 2020; Watt et al., 2012). Eine zentrale Erkenntnis - neben der oben beispielhaft skizzierten Be- deutung von Studienwahlmotiven für Studien- und Berufserfolgsmaße - ist dabei u.a., dass sich Studentinnen und Studenten verschiedener Lehramtsstudiengänge und Länder in ihren Studienwahlmotivationen teilweise deutlich unterscheiden (Lin et al., 2012; Retelsdorf \& Möller, 2012; Rutsch et al., 2020; Watt et al., 2012). Damit stellt sich auch die Frage, warum länder- oder studiengangspezifische Gegebenheiten eines Lehramtsstudiums für bestimmte Personen besonders attraktiv sind, und warum sich andere Personen für andere Studiengänge entscheiden. Zur Beantwortung dieser Frage erscheinen also auch studiengangübergreifende Untersuchungen wichtig, die Studienwahlmotive von Lehramtsstudentinnen und -studenten nicht nur absolut und für sich, sondern vor allem auch im Vergleich mit Studentinnen und Studenten anderer Studiengänge theoretisch und empirisch diskutieren. Hierzu liegen bisher jedoch nur vereinzelte Studien vor (z.B. Heublein et al., 2017; Neugebauer, 2013), die allerdings Studienwahlmotive in der Regel allein auf Basis von Einzelitems und nicht mittels standardisierter und reliabler Fragebogenskalen erfassen und die daher nur bedingt wissenschaftlich belastbar erscheinen. Diesem Desideratum widmet sich der vorliegende Beitrag.

\section{Theorie: Konzeption, Klassifikation und Erfassung von Studienwahl- motiven für ein Lehramtsstudium}

In der Literatur wird zwischen den Begriffen „Studienwahlmotive" und "Studienwahlmotivation" oft nicht konsequent unterschieden, vermutlich auch, weil Motivation und Motive eng verwandte Konstrukte darstellen. Die $\mathrm{Mo}$ tivation einer Person wird als „die aktivierende Ausrichtung des momentanen Lebensvollzugs auf einen positiv bewerteten Zielzustand bzw. auf das Vermeiden eines negativ bewerteten Zustandes" (Rheinberg \& Vollmeyer, 2018, S.17) definiert, wohingegen unter Motiven relativ zeitstabile Präferenzen einer Person für bestimmte Anreizklassen verstanden werden (Rheinberg \& Vollmeyer, 2018). Sowohl Motivation als auch Motive einer Person 
sind dabei hypothetische Konstrukte, die nicht unmittelbar beobachtet werden können. Theoretisch werden Motive als ,einzelne, isolierte Beweggründe menschlicher Verhaltensbereitschaft" (Becker, 2019, S.20) aufgefasst, aus deren Wechselwirkung mit situativen Anreizen die Motivation und daraus resultierende Handlungen einer Person (zumindest teilweise) erklärt werden können (Heckhausen \& Heckhausen, 2018). Ausgehend von diesen Überlegungen definieren wir Studienwahlmotive als relativ zeitstabile Beweggründe einer Person für die Wahl ihres Studiums. Im Folgenden sollen nun solche Studienwahlmotive identifiziert werden, aus deren Zusammenspiel sich die Motivation für die Wahl eines Studiengangs möglichst gut erklären lässt.

Klassifikationen verschiedener Studienwahlmotive für ein Lehramtsstudium greifen in der Regel auf allgemeine Motivationstheorien zurück (z. B. Heckhausen \& Heckhausen, 2018; Rheinberg \& Vollmeyer, 2018). Ein Ansatz ist dabei die Unterscheidung zwischen intrinsischer, also in der Sache (bzw. den zugehörigen Handlungen) selbst liegender, und extrinsischer Motivation, bei der die Entscheidung für z. B. eine Handlung aufgrund von außerhalb dieser Handlung liegender Ziele getroffen wird (z. B. Ryan \& Deci, 2000). Typische Beispiele für intrinsische Motive zur Aufnahme eines Studiums sind z. B. fachliches Interesse an den Studieninhalten, für extrinsische Motive z.B. die Aussicht auf ein sicheres bzw. hohes Einkommen im späteren Beruf (für Beispielitems zur Erhebung dieser und der im Folgenden genannten Studienwahlmotive siehe Tabelle 3). Neben der Unterscheidung von intrinsischen und extrinsischen Studienwahlmotiven identifizieren viele - insbesondere auch qualitative - Studien zusätzlich altruistische Studienwahlmotive für die Wahl eines Lehramtsstudiums, wie z.B. den Wunsch, einen Beitrag für die Gesellschaft zu leisten oder anderen Menschen zu helfen. Diese altruistischen Motive können sogar stärker als intrinsische oder extrinsische Studienwahlmotive ausgeprägt sein (Brookhart \& Freeman, 1992; Fray \& Gore, 2018).

Ein weiterer Ansatz zur Klassifikation von Studienwahlmotiven stellt ein Rückgriff auf Erwartungs-Wert-Modelle dar. Bei diesen wird angenommen, dass die Motivation von Individuen für eine Aufgabe durch deren Erwartungen, wie erfolgreich sie diese Aufgabe bearbeiten können, und den Wert, den jene dieser Aufgabe zumessen, erklärt werden kann (z. B. Eccles \& Wigfield, 2002; Wigfield et al., 2015). Der Wert der Aufgabe ergibt sich dabei aus dem Interesse bzw. der Freude an der Bearbeitung der Aufgabe (interest-enjoyment value), der persönlichen Bedeutung bzw. Wichtigkeit, die Aufgabe gut zu bearbeiten (attainment value), der Nützlichkeit der Bearbeitung der Aufgabe für andere aktuelle und zukünftige Ziele (utility value) sowie den Kosten (relative cost), die die Bearbeitung der Aufgabe mit sich bringt (z. B. in Form von benötigtem Arbeitsaufwand oder Versagensängsten, Eccles \& Wigfield, 2002). Auch die oben genannten intrinsischen, extrinsischen und altruistischen Studienwahlmotive lassen sich anhand dieser Werte klassifizieren: Der Wert intrinsischer Studienwahlmotive, wie z.B. fachlichem Interesse, ergibt sich aus dem Interesse bzw. der Freude an der Auseinandersetzung mit den Studieninhalten (interest-enjoyment value), der Wert extrinsischer Studienwahlmotive, wie z.B. der Aussicht auf ein sicheres bzw. hohes Einkommen, liegt in der persönlichen Nützlichkeit (utility value), die das Studium für dieses Einkommen hat, und der Wert altruistischer Studienwahlmotive, wie dem Wunsch, einen Beitrag für die Gesellschaft zu leisten oder anderen Menschen

Tabelle 1. Übersicht über theoretisch und empirisch identifizierte und untersuchte Studienwahlmotive und ihre motivationstheoretische Zuordnung

\begin{tabular}{|c|c|c|}
\hline $\begin{array}{l}\text { Zuordnung im Erwartungs-Wert- } \\
\text { Modell }\end{array}$ & $\begin{array}{l}\text { Beispiele typischer Studienwahlmotive } \\
\text { (z. B. Fray \& Gore, 2018; Heublein et al., 2017; } \\
\text { Pohlmann \& Möller, 2010; Watt et al., 2012) }\end{array}$ & $\begin{array}{l}\text { Zuordnung zu intrinsischen, extrinsischen } \\
\text { oder altruistischen Motiven }\end{array}$ \\
\hline Erwartungskomponente & $\begin{array}{l}\text { Fähigkeitsüberzeugungen } \\
\text { (bzgl. der Studienfächer, } \\
\text { bzgl. eigener Lehrfähigkeiten) }\end{array}$ & - \\
\hline $\begin{array}{l}\text { Interessen } \\
\text { (interest value) }\end{array}$ & $\begin{array}{c}\text { fachliches Interesse, } \\
\text { pädagogisches Interesse, } \\
\text { berufliches Interesse (fester Berufswunsch) }\end{array}$ & intrinsisch \\
\hline $\begin{array}{l}\text { Gesellschaftlicher Wert } \\
\text { (attainment value) }\end{array}$ & $\begin{array}{l}\text { einen gesellschaftlichen Beitrag leisten, } \\
\text { anderen Menschen helfen }\end{array}$ & altruistisch \\
\hline $\begin{array}{l}\text { Persönliche Nützlichkeit } \\
\text { (utility value) }\end{array}$ & $\begin{array}{l}\text { hohes bzw. sicheres Einkommen, } \\
\text { Vereinbarkeit von Familie und Beruf }\end{array}$ & extrinsisch \\
\hline $\begin{array}{l}\text { Kosten } \\
\text { (relative cost) }\end{array}$ & $\begin{array}{l}\text { geringe Schwierigkeit des Studiums, } \\
\text { soziale Einflüsse (Rat von anderen), } \\
\text { Nähe zum Wohnort }\end{array}$ & - \\
\hline
\end{tabular}


zu helfen, kann in der Wichtigkeit (attainment value), die diesen Aufgaben zugesprochen wird, gesehen werden (vgl. Watt et al., 2012).

In Tabelle 1 ist eine Übersicht über diese Zuordnungen der beiden möglichen Klassifikationsansätze gegeben. Man sieht hier zudem, dass sich einige weitere theoretisch und empirisch identifizierte und untersuchte Studienwahlmotive im Erwartungs-Wert-Modell einordnen lassen. Dies betrifft insbesondere Studienwahlmotive wie die geringe Schwierigkeit des Studiums, soziale Einflüsse (z.B. den Rat von anderen zur Aufnahme des Studiums) oder die Nähe des Studienorts zum Wohnort, die hier als Kosten (bzw. Motiven zur Senkung von Kosten) eingeordnet werden und sich nicht eindeutig als intrinsische oder extrinsische Studienwahlmotive identifizieren lassen. Schließlich können auf der Ebene der Erwartungen persönliche Fähigkeitsüberzeugungen, z.B. mit Blick auf die gewählten Studienfächer aber auch den späteren Beruf, als eigenständiger Faktor identifiziert werden (Pohlmann \& Möller, 2010; Watt et al., 2012). Diese Klassifikation ist z. B. in aktuell - insbesondere im deutschsprachigen Raum - gängigen Instrumenten, wie dem ,Fragebogen zur Erfassung der Motivation für die Wahl des Lehramtsstudiums" (FEMOLA Pohlmann \& Möller, 2010) und der „FITChoice scale" (Watt et al., 2012), operationalisiert.

Die in Tabelle 1 vorgeschlagene Zuordnung zeigt, dass sich theoretisch und empirisch identifizierte und untersuchte Studienwahlmotive für ein Lehramtsstudium mithilfe des Erwartungs-Wert-Modells gut theoretisch beschrieben lassen. Insbesondere lassen sich damit auch diejenigen Studienwahlmotive, die hier den Kosten zugeordnet sind, theoretisch einordnen. Zudem erlaubt das Erwartungs-Wert-Modell eine Unterscheidung zwischen intrinsischen Studienwahlmotiven und Fähigkeitsüberzeugungen, die sonst oftmals als gemeinsamer intrinsischer Faktor aufgefasst werden (z. B. Hanfstingl \& Mayr, 2007; Heublein et al., 2017), die sich aber tatsächlich auch empirisch als unterschiedliche Faktoren herausstellen (Pohlmann \& Möller, 2010; Rutsch et al., 2020).

Vollumfänglich unproblematisch ist aber auch diese theoretische Einordnung von Studienwahlmotiven für spätere empirische Operationalisierungen nicht. So ergibt eine Betrachtung der in Tabelle 1 aufgeführten Studienwahlmotive „pädagogisches Interesse“, das in der Regel über Interesse bzw. Freude an der Arbeit mit Kindern und Jugendlichen operationalisiert ist (Pohlmann \& Möller, 2010; Watt et al., 2012), und „Fähigkeitsüberzeugung bzgl. eigener Lehrfähigkeiten“, die z.B. über die Überzeugung, gut erklären zu können, operationalisiert wird (Pohlmann \& Möller, 2010): Diese Studienwahlmotive sind für Lehramtsstudiengänge zwar einerseits hochrelevant, da ein Lehramtsstudium, wie kaum ein anderer Studiengang, auf einen bestimmten Beruf ausgerichtet ist (Spinath et al.,
2005) und dementsprechend bei der Studienwahl schon die Berufswahl mitgedacht ist. Konzeptionell kann genau dies andererseits jedoch problematisch sein, da erstens pädagogisches Interesse ein intrinsisches Motiv für die Berufswahl ist, aus dem sich aber nicht notwendigerweise eine intrinsische Motivation für das Studium ergibt, und da zweitens aus einer berufsbezogenen Fähigkeitsüberzeugung nicht unbedingt eine studienbezogene Fähigkeitsüberzeugung folgt. Für eine Erhebung von Studienwahlmotiven von Bewerberinnen und Bewerber auf Nicht-Lehramtsstudiengänge scheinen diese Motive entsprechend eher ungeeignet.

\section{Empirischer Forschungsstand}

\section{Personenzentrierte Erfassung von Studienwahlmotiven durch Motivprofile}

In aktuellen Studien ist die Betrachtung von verschiedenen Motivprofilen von Lehramtsstudentinnen und -studenten stärker in den Fokus gerückt (z.B. Biermann et al., 2019; Billich-Knapp et al., 2012; Dörrenbächer-Ulrich et al., 2019; König et al., 2018). Die dahinterliegende - auch empirisch fundierte (z.B. Sinclair, 2008) - Idee ist dabei, dass für die Studienwahlentscheidung nicht einzelne Motive, sondern vielmehr das Zusammenspiel mehrerer solcher Studienwahlmotive ausschlaggebend ist. Dieser Ansatz entspricht auch den oben ausgeführten theoretischen Überlegungen, dass die Motivation für die Wahl eines Studiums durch die Wechselwirkung verschiedener Motive und situativer Anreize erklärt werden kann. Dadurch kann die Betrachtung verschiedener Motivprofile als personenzentrierter Ansatz verstanden werden, bei dem Gruppen von Personen mit ähnlichen motivationalen Merkmalsausprägungen im Vordergrund stehen (vgl. König et al., 2018) - im Gegensatz zu variablenzentrierten Ansätzen, die sich eher auf die Untersuchung einzelner Motive fokussieren.

Clusteranalysen zur Bestimmung solcher Motivprofile identifizieren dabei in den meisten Fällen drei, teilweise aber noch mehr solcher Profile (siehe König et al., 2018 für einen Überblick). Lösungen mit drei Clustern ergeben dabei oft (1) ein vorrangig intrinsisch motiviertes Profil, in dem fachliche und pädagogische Interessen sowie Fähigkeitsüberzeugungen vergleichsweise hoch und die Motive zur persönliche Nützlichkeit und Kosten vergleichsweise niedrig ausgeprägt sind, (2) ein vorrangig extrinsisch motiviertes Profil, in dem die Motive zur persönliche Nützlichkeit und Kosten vergleichsweise hoch und fachliche und pädagogische Interessen sowie Fähigkeitsüberzeugungen vergleichsweise niedrig ausgeprägt sind und (3) ein ausgewogen (hoch) motiviertes Profil, in dem alle Studienwahl- 
motive vergleichsweise hoch ausfallen (z. B. Biermann et al., 2019; Billich-Knapp et al., 2012; Kiel et al., 2015; König et al., 2018; Thomson et al., 2012). Dörrenbächer-Ulrich et al. (2019) erhalten eine 4-Cluster-Lösung, die zusätzlich zu diesen drei Motivprofilen noch ein ausgewogen niedrig motiviertes Profil identifiziert.

Die Bedeutung solcher Motivprofile ergibt sich insbesondere durch deren Zusammenhänge mit Variablen mit Bezug zu Studienerfolg und professionellen Kompetenzen. Insbesondere stellen sich dabei Studentinnen und Studenten aus dem vorrangig extrinsisch motivierten Profil als oftmals benannte „Risikogruppe“ heraus, die eine geringer ausgeprägte Leistungsmotivation und Lernzielorientierung (Billich-Knapp et al., 2012; König et al., 2018) sowie eine geringere Lehrerselbstwirksamkeitserwartung (Dörrenbächer-Ulrich et al., 2019) aufweisen. Biermann et al. (2019) konnten zudem zeigen, dass in einem solchen Profil die selbstberichtete Nutzung von Lerngelegenheiten (pädagogische Handlungssituationen planen, pädagogische Handlungssituationen durchführen, Theorien auf Situationen beziehen, mit Situationen analytisch-reflexiv umgehen) und die selbsteingeschätzte Kompetenz hinsichtlich Unterrichtsplanung sowie Durchführung von Unterricht und Diagnostik geringer (die selbsteingeschätzte Kompetenz hinsichtlich Disziplinproblemen jedoch höher) ausfällt als im vorrangig intrinsisch und ausgewogen motivierten Profil. Der Anteil der Studentinnen und Studenten, die dem vorrangig extrinsisch motivierten Profil zugeordnet werden, bewegt sich dabei in den hier näher beschriebenen Studien zwischen $8.7 \%$ (König et al., 2018) und 23.2\% (Dörrenbächer-Ulrich et al., 2019).

\section{Länder- und Studiengangunterschiede}

Viele der aktuellen Studien zu Studienwahlmotiven befassen sich ausschließlich mit Lehramtsstudentinnen und -studenten. Dabei zeigt sich, dass absolut gesehen im deutschsprachigen Raum über verschiedene Lehramtsstudiengänge hinweg pädagogische und fachliche Interessen die am höchsten ausgeprägten Studienwahlmotive sind, gefolgt von Fähigkeitsüberzeugungen und gesellschaftlichen Werten, die etwas höher ausfallen als die Studienwahlmotive zur persönlichen Nützlichkeit, wobei die geringe Schwierigkeit des Lehramtsstudiums die geringste Zustimmung erhält (Biermann et al., 2019; Dörrenbächer-Ulrich et al., 2019; König et al., 2013, 2018; Micknass et al., 2019; Pohlmann \& Möller, 2010; Retelsdorf \& Möller, 2012).

Innerhalb dieses deutschsprachigen Raums finden sich dabei insgesamt kaum Unterschiede in den Studienwahlmotiven von deutschen und österreichischen (König et al., 2018) sowie deutschen und schweizer (König et al., 2013) Lehramtsstudentinnen und -studenten. Im Vergleich mit anderen Ländern zeigen sich deutsche Lehramtsstudentinnen und -studenten jedoch bspw. stärker extrinsisch motiviert als finnische Lehramtsstudierende (Goller et al., 2019) und der intrinsische Wert des Lehrerinnen- und Lehrerberufs sowie der Fähigkeitsüberzeugungen bzgl. Lehrfähigkeiten fällt bei deutschen Lehramtsstudentinnen und -studenten geringer aus als bei australischen und amerikanischen zukünftigen Lehrkräften (Watt et al., 2012). Gesellschaftliche Werte sind bei amerikanischen Lehramtsstudentinnen und -studenten sowohl im Vergleich zu anderen Studienwahlmotiven (Lin et al., 2012; ebenso wie bei türkischen, Kılınç et al., 2012) als auch im Vergleich mit australischen, deutschen und norwegischen Lehramtsstudentinnen und -studenten (Watt et al., 2012) am höchsten ausgeprägt. In China überwiegen Motive zu gesellschaftlichem Wert und persönlicher Nützlichkeit (Lin et al., 2012; siehe Fray \& Gore, 2018 für weitere Länderunterschiede). Solche Unterschiede werden durch sich unterscheidende Arbeitsmarktbedingungen (Neugebauer, 2015) sowie unterschiedliche kulturelle, gesellschaftliche und auch religiöse Einflüsse (Afrianto, 2014; Azman, 2013) in den verschiedenen Ländern erklärt und lassen zudem Rückschlüsse z.B. auf kulturelle und gesellschaftliche Unterschiede mit Blick auf die Bedeutung (bzw. die von Studentinnen und Studenten wahrgenommen Werte) eines Lehramtsstudiums in den verschiedenen Ländern zu.

Unterschiede in den Studienwahlmotiven von Lehramtsstudentinnen und -studenten finden sich auch mit Blick auf unterschiedliche Fächer (Fischer et al., 2019; Micknass et al., 2019; Watt et al., 2017) und unterschiedliche Schulformen. So sind z.B. fachliche Interessen bei Studentinnen und -studenten des gymnasialen Lehramts stärker ausgeprägt als im Realschullehramt (Pohlmann \& Möller, 2010; Retelsdorf \& Möller, 2012) und bei Studentinnen und Studenten des Realschullehramts wiederum stärker als im Grundschullehramt (Rutsch et al., 2020). Beim pädagogischen Interesse dreht sich diese Reihenfolge um (Retelsdorf \& Möller, 2012). Derartige Ergebnisse zeigen, dass studiengangübergreifende Vergleiche $\mathrm{zu}$ einem besseren Verständnis von Studienwahlentscheidungen beitragen können und dass diese damit auch von praktischer Relevanz für die Konzeption von universitären Lehrveranstaltungen sein können, da die Ergebnisse Hinweise darauf geben, wie Studentinnen und Studenten verschiedener Studiengänge motiviert sind bzw. werden können.

Vergleiche, die über das Lehramt hinausgehende Studiengänge miteinbeziehen, sind jedoch kaum zu finden (siehe z. B. Heublein et al., 2017; Neugebauer, 2013; Windolf, 1992 für Ausnahmen). Ein Grund hierfür mag auch sein, dass bisher kein einheitliches Instrument existiert, dass die oben ausgeführte Unterscheidung von Studienwahlmotiven auf Basis des Erwartungs-Wert-Modells 
studiengangübergreifend auch für Nicht-Lehramtsstudiengänge operationalisiert. Studien zu studiengangübergreifenden Vergleichen von Studienwahlmotiven beruhen in der Regel auf Einzelitems und liefern auf dieser Basis weitere Interpretationsansätze (z.B. Heublein et al., 2017; Neugebauer, 2013). Z. B. zeigt sich, dass fachliche und wissenschaftliche Interessen bei Studentinnen und Studenten aus Nicht-Lehramtsstudiengängen im Mittel höher ausgeprägt sind als bei Studentinnen und Studenten aus Lehramtsstudiengängen, wohingegen bei Studentinnen und Studenten aus Lehramtsstudiengängen gesellschaftliche Werte, berufliche Sicherheit und eine gute Vereinbarkeit von Familie und Beruf vergleichsweise höher ausgeprägte Studienwahlmotive sind (Neugebauer, 2013).

\section{Zusammenfassung}

Studienwahlmotive von Lehramtsstudentinnen und -studenten sind insbesondere mit Blick auf ein grundsätzliches Interesse an Lehrerinnen- und Lehrerpersönlichkeiten vielfach untersucht worden und haben eine prädiktive Bedeutung für Studien- und Berufserfolg. Einige Studien berücksichtigen zudem, dass für die Studienwahl in der Regel nicht nur einzelne Studienwahlmotive, sondern vielmehr Bündel von solchen Motiven entscheidend sind, und identifizieren auf dieser Basis entsprechende Motivprofile. Studiengangübergreifende Untersuchungen, die hierbei auch Studentinnen und Studenten aus Nicht-Lehramtsstudiengängen miteinbeziehen, können zur besseren Einordnung empirischer Ergebnisse beitragen, sind aber äußerst selten und beruhen in der Regel auf Einzelitems. Im Folgenden sollen also Studienwahlmotive auf der Grundlage des ErwartungsWert-Modells studiengangübergreifend (sowohl variablenzentriert als auch profilanalytisch) untersucht und Er- kenntnisgewinne aus diesen Studiengangvergleichen diskutiert werden.

\section{Forschungsfragen}

Aus dem vorangegangenen Abschnitt ergeben sich vier Forschungsfragen, die im Folgenden untersucht werden:

1. Lassen sich die auf der Basis des Erwartungs-WertModells für Lehramtsstudentinnen und -studenten entwickelte Klassifikationen von Studienwahlmotiven auf andere Studiengänge übertragen und reliabel erfassen?

2. Unterscheiden sich Bewerberinnen und Bewerber auf ein Lehramtsstudium in diesen Studienwahlmotiven von Bewerberinnen und Bewerbern auf andere Studiengänge?

3. Welche Motivprofile lassen sich studiengangübergreifend identifizieren?

4. Wie verorten sich Bewerberinnen und Bewerber auf verschiedene Studiengänge innerhalb dieser Motivprofile?

\section{Methode}

\section{Stichprobe}

Die Leuphana Universität Lüneburg führt jährlich im Rahmen des Bewerbungsverfahrens für diejenigen Bewerberinnen und Bewerber auf ein Studium, die nicht direkt über gesetzlich vorgeschrieben Sonderquoten (bspw. Bestenquoten) zum Studium zugelassen werden, so genannte Auswahltage durch. Durch eine erfolgreiche Teilnahme an diesen können die Bewerberinnen und Bewerber ihre Chancen auf Zulassung erhöhen. Im Sommer 2019 haben an diesen Auswahltagen 690 Bewerberinnen und Bewerber auf einen Studienplatz (davon 537 weiblich, 143 männ-

Tabelle 2. Übersicht über die Stichprobe

\begin{tabular}{lccccccccc}
\hline & \multicolumn{3}{c}{ Stichprobe } & \multicolumn{3}{c}{ Auswahlverfahren } & & \multicolumn{2}{c}{ Immatrikuliert } \\
\hline $\begin{array}{l}\text { Studiengang- } \\
\text { gruppe }\end{array}$ & $N$ & HZB & $w$ & $N$ & HZB & $w$ & $N$ & HZB & $w$ \\
\hline Lehramt & 252 & 2.32 & $83.3 \%$ & 554 & 2.41 & $77.4 \%$ & 391 & 2.31 & $72.6 \%$ \\
$\begin{array}{l}\text { Nachhaltig- } \\
\text { keit }\end{array}$ & 157 & 1.72 & $80.9 \%$ & 343 & 1.74 & $79.3 \%$ & 213 & 1.58 & $82.2 \%$ \\
Kultur & 209 & 2.26 & $76.6 \%$ & 775 & 2.26 & $71.9 \%$ & 365 & 2.08 & $71.0 \%$ \\
Wirtschaft & 72 & 2.33 & $55.6 \%$ & 550 & 2.45 & $50.9 \%$ & 401 & 2.25 & $51.4 \%$ \\
\hline Gesamt & 690 & 2.17 & $77.8 \%$ & 2222 & 2.27 & $69.2 \%$ & 1370 & 2.12 & $67.4 \%$ \\
\hline
\end{tabular}

Anmerkung: Anzahlen (N), Mittelwerte der Hochschulzugangsberechtigungsnoten (HZB) und prozentuale Anteile der weiblichen Bewerberinnen $(w)$ in den jeweiligen Studienganggruppen in der Stichprobe im Vergleich zu der Gesamtheit der am Auswahlverfahren teilnehmenden Bewerberinnen und Bewerber und der später Immatrikulierten. 
lich, 1 divers, 9 unbekannt) im Alter zwischen 17 und 37 Jahren $(M=20, S D=2.07)$ an einer Befragung zur Erfassung von Studienwahlmotiven freiwillig teilgenommen (Rücklaufquote: $40 \%$ ).

Die 690 Bewerberinnen und Bewerber haben sich insgesamt auf 13 verschiedene Studiengänge beworben. Diese lassen sich vier grundlegend verschiedenen Studienganggruppen zuordnen:

- Die Studienganggruppe Lehramt umfasst die Studiengänge „Lehren und Lernen“ (Grund-Haupt-RealschulLehramt, $N=209)$, „Wirtschaftspädagogik“ $(N=30)$ und "Sozialpädagogik" $(N=13)$.

- Die Studienganggruppe Nachhaltigkeit umfasst die Studiengänge "Global Environmental and Sustainability Studies“ ( $N=35)$, „Umweltwissenschaften“ $(N=88)$ und ,Studium Individuale“ $(N=34)$.

- Die Studienganggruppe Kultur umfasst die Studiengänge „Digital Media“ $(N=35)$, „Kulturwissenschaften“ $(N=84)$, ,Politikwissenschaften“ $(N=19)$ und „Psychologie“ $(N=71)$.

- Die Studienganggruppe Wirtschaft umfasst die Studiengänge „Betriebswirtschaftslehre“ $(N=24)$, „International Business Administration and Entrepreneurship" $(N=32)$ und „Rechtswissenschaften“ $(N=16)$.

Die Stichprobe ist mit Blick auf die durchschnittliche Hochschulzugangsberechtigungsnote (Abiturnote) und die Geschlechterverteilung zur Gesamtheit der am Auswahlverfahren teilnehmenden Bewerberinnen und Bewerber sowie zur im WS 2019 / 20 im ersten Semester immatrikulierten Studierenden ähnlich, wobei weibliche Bewerberinnen in der Stichprobe leicht überrepräsentiert sind (siehe Tabelle 2).

\section{Instrumente}

Für die Erhebung von Studienwahlmotiven wurde auf der Basis bestehender Instrumente ein Fragebogen zusammengestellt, der zur Erwartungskomponente und zu jeder der vier Wertkomponenten des Erwartungs-WertModells jeweils mindestens ein in Tabelle 1 aufgeführtes Studienwahlmotiv für einen studiengangübergreifenden Einsatz operationalisiert: In der Erwartungskomponente durch Items zu fachlichen Fähigkeitsüberzeugungen hinsichtlich der gewählten Studienfächer (in Anlehnung an das Selbstkonzept Fach nach Retelsdorf et al., 2014), in den Wertkomponenten durch Items zu fachlichem Interesse (durch Adaption der entsprechenden Skala des „Fragebogen zur Erfassung der Motivation für die Wahl des Lehramtsstudiums" (FEMOLA), Pohlmann \& Möller, 2010), zu beruflichem Interesse (Eigenentwicklung), zum gesellschaftlichen Wert des Studiums (durch Adap- tion der Skala „Make social contribution“ der FIT-Choice scale, Watt et al., 2012), zur persönlichen Nützlichkeit, zur geringen Schwierigkeit des Studiums, zu sozialen Einflüssen (durch Adaption der entsprechenden Skalen des FEMOLA, Pohlmann \& Möller, 2010) sowie zur Nähe zum Wohnort (Eigenentwicklung, siehe Tabelle 3 für die genauen Itemformulierungen). Die Adaptionen der Skalen aus dem FEMOLA und der FIT-Choice scale erfolgten, damit der Fragebogen auch für Bewerberinnen und Bewerber auf Nicht-Lehramtsstudiengänge sinnvoll bearbeitbar war. In den meisten Fällen reichten hier minimale Umformulierungen der Originalitems aus (indem z.B. „als Lehrer*in“ durch „später“ oder „Lehramtsstudium“ durch „Studium“ ersetzt wurde). Sämtliche Items/Skalen wurden papierbasiert mithilfe 5-stufiger Likert-Skalen $(1=$,trifft gar nicht zu“; 5 = „trifft völlig $\mathrm{zu}$ “) eingesetzt.

\section{Auswertung}

Zur Beantwortung von Forschungsfrage 1 wurden explorative (mit SPSS 26) und konfirmatorische (mit dem RPacket lavaan (Rosseel, 2019) für ordinal-skalierte Variablen (siehe Mair, 2018 für das genaue Vorgehen)) Faktorenanalysen durchgeführt.

Zur Überprüfung von Studiengangunterschieden (Forschungsfrage 2) erfolgten Varianzanalysen (in SPSS 26) für die vier benannten Studienganggruppen.

Zur Beantwortung von Forschungsfrage 3 wurden (in SPSS 26) $K$-Means Clusteranalysen für die festgesetzten Clusteranzahlen $K=1, \ldots, 6$ berechnet. Da so erhaltene Clusterlösungen potenziell von der Wahl der Startwerte abhängen, wurden diese Analysen mit unterschiedlichen Startwerten (z.B. Clusterzentren von anderen Clusterverfahren aus SPSS 26, Sortierung der Datenmatrix nach verschiedenen Spalten) wiederholt (vgl. Schendera, 2010). Zur Bewertung der so erhaltenen Clusterlösungen wurde für jede Lösung die Streuungsquadratsumme in den Clustern $S Q_{i n}(K)$, d.h. die Summe der Quadrate der Distanzen zum jeweiligen Clusterzentrum, berechnet. Für jedes $K=1, \ldots, 6$ wurde dann die Lösung mit der minimalen Streuungsquadratsumme ausgewählt. Zur weiteren Bewertung dieser Clusterlösungen wurde für jede Clusteranzahl $K=1, \ldots, 6$ die durch die zugehörige Clusterlösung erklärte Streuung $\eta_{K}^{2}$, die proportionale Fehlerverbesserung im Vergleich zur Vorgängerlösung $P R E_{K}$ und jeweils die maximale $F$-Statistik $F_{\max K}$ der Clusterlösungen berechnet (siehe Bacher et al., 2010 für das genaue Vorgehen).

Für Forschungsfrage 4 wurde schließlich untersucht, wie sich die verschiedenen Studienganggruppen auf die Cluster verteilen. 


\section{Ergebnisse}

\section{Ergebnisse zu Forschungsfrage 1}

Eine explorative Hauptkomponentenanalyse des adaptierten Fragebogens mit Varimax-Rotation liefert sieben Fakto- ren mit Eigenwerten größer als 1.0 (siehe Tabelle 3). Diese bestätigen die theoretisch angenommene Faktorenstruktur fast vollständig (lediglich „Soziale Einflüsse“ und „Nähe zum Wohnort" fallen zu einem Faktor zusammen) und erklären $64 \%$ der Gesamtvarianz. Der Screeplot (Abbildung 1)

Tabelle 3. Ergebnisse der explorativen Faktorenanalyse

\begin{tabular}{|c|c|c|c|c|c|c|c|}
\hline \multicolumn{8}{|l|}{ Rotierte Komponentenmatrix } \\
\hline \multirow[t]{2}{*}{ Ich habe mich für meinen Studiengang beworben, ... } & \multicolumn{7}{|c|}{ Komponente } \\
\hline & 1 & 2 & 3 & 4 & 5 & 6 & 7 \\
\hline \multicolumn{8}{|l|}{ Erwartungskomponente (Fachliche Fähigkeitsüberzeugung) } \\
\hline weil mir diese Studienfächer besonders liegen. & & & & & & & .844 \\
\hline weil ich in diesen Studienfächern ziemlich fit bin. & & & & & & & .819 \\
\hline \multicolumn{8}{|l|}{ Wert: Interesse 1 (Fachliches Interesse) } \\
\hline weil mich die Methoden, Theorien und Erkenntnisse dieses Fachs besonders interessieren. & & & .750 & & & & \\
\hline weil ich mich gern mit den Inhalten dieser Fächer beschäftige. & & & .720 & & & & \\
\hline weil ich viel in diesen Studienfächern dazu lernen möchte. & & & .671 & -.203 & & & \\
\hline weil ich die Inhalte der Fächer interessant finde. & & & .616 & & & & .228 \\
\hline
\end{tabular}

Wert: Interesse 2 (Berufliches Interesse)

um später meinen Wunschberuf ausüben zu können.

weil ich mich für einen bestimmten Beruf interessiere.

.286

.798

Wert: Gesellschaftlicher Wert des Studiums

um später einen wertvollen sozialen Beitrag leisten zu können.

um später etwas Nützliches für die Gesellschaft zu tun.

damit ich später der Gesellschaft etwas zurückgeben kann. $\quad .814$

um anderen Menschen zu helfen.

$\begin{array}{ll}\text { Wert: Persönliche Nützlichkeit } & .866 \\ \text { damit ich später finanziell abgesichert bin. } & .848 \\ \text { damit ich später gut verdiene. } & .802 \\ \text { damit ich später regelmäßig ein festes Gehalt bekomme. } & .729 \\ \text { um einen angesehenen Beruf zu bekommen. } & .697 \\ \text { weil ich so später Familie und Beruf gut vereinbaren kann. } & .648 \\ \text { weil ich neben dem Beruf auch noch Zeit für Familie, Freunde und Hobbys haben will. }\end{array}$

Wert: Kosten 1 (geringe Schwierigkeit des Studiums)

$\begin{array}{lr}\text { weil dieser Studiengang nicht so anstrengend ist. } & .822\end{array}$

weil dieser Studiengang leichter ist als andere Studiengänge.

weil ich denke, dass ich in diesem Studiengang ohne große Mühe durchkomme. $\quad .626$

Wert: Kosten 2 (Soziale Einflüsse und Nähe zum Wohnort)

weil ich so in der Nähe meines Wohnorts studieren kann.

weil ich in Lüneburg studieren möchte. $\quad .644$

weil ich denke, dass meine Familie und meine Freunde diesen Studiengang am besten $\quad .259 \quad 639$

geeignet für mich halten.

$.305 \quad .569$

weil mir von Freunden und Bekannten zu diesem Studiengang geraten wurde.

Anmerkung: Faktorladungen unter 0.2 sind nicht aufgeführt. Faktor 1: persönliche Nützlichkeit (abgekürzt als „nützlich“); Faktor 2: gesellschaftlicher Wert des Studiums („Gesellschaft“); Faktor 3: fachliches Interesse („Fach“); Faktor 4: geringe Schwierigkeit des Studiums (,einfach“); Faktor 5: Soziale Einflüsse und Nähe zum Wohnort; Faktor 6: berufliches Interesse (,Beruf“); Faktor 7: fachliche Fähigkeitsüberzeugung (,Fähigkeit“). 


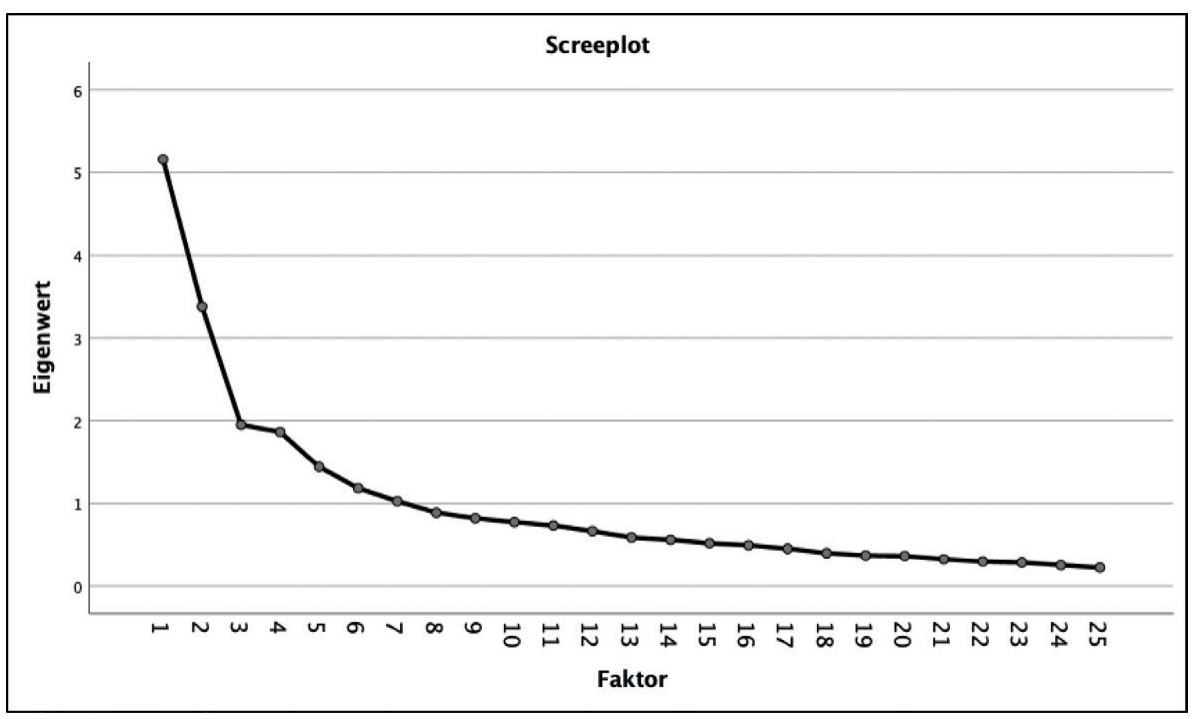

Abbildung 1. Screeplot zur explorativen Faktorenanalyse.

Tabelle 4. Deskriptive Werte

\begin{tabular}{|c|c|c|c|c|c|c|c|}
\hline Skala & $\alpha$ & Min & $\operatorname{Max}$ & $M$ & $S D$ & Schiefe & Kurtosis \\
\hline Fähigkeit & .72 & 1.50 & 5.00 & 3.93 & 0.61 & -0.39 & 0.45 \\
\hline Fach & .69 & 3.00 & 5.00 & 4.63 & 0.41 & -1.22 & 1.25 \\
\hline Beruf & .76 & 1.00 & 5.00 & 4.17 & 0.94 & -1.12 & 0.44 \\
\hline Gesellschaft & .85 & 1.00 & 5.00 & 4.22 & 0.75 & -1.21 & 1.50 \\
\hline Nützlich & .88 & 1.00 & 5.00 & 3.30 & 0.91 & -0.45 & -0.32 \\
\hline Einfach & .71 & 1.00 & 4.33 & 1.85 & 0.68 & 0.60 & -0.16 \\
\hline $\begin{array}{l}\text { Soziale Einflüsse } \\
\& \text { Wohnort }\end{array}$ & .58 & 1.00 & 5.00 & 2.91 & 0.88 & -0.03 & -0.64 \\
\hline
\end{tabular}

deutet zudem auf eine mögliche Zwei-Faktoren-Lösung hin. Eine zusätzliche konfirmatorische Faktorenanalyse zeigt jedoch, dass die Sieben-Faktoren-Lösung wesentlich bessere Modell-Fit-Werte (Standardisierte $\chi^{2}(254)=$ 788.759, $p<0.001, C F I=0.979, T L I=0.975, R M S E A=$ $0.058, S R M R=0.068)$ aufweist als die Zwei-FaktorenLösung (Standardisierte $\chi^{2}(274)=3923.621, p<0.001$, $C F I=0.857, T L I=0.843, R M S E A=0.146, S R M R=0.137)$. Die internen Konsistenzen der so erhaltenen Skalen sind mit einer Ausnahme im akzeptablen bis sehr guten Bereich (siehe Tabelle 4). Der Faktor „Soziale Einflüsse und Nähe zum Wohnort" wird aufgrund seiner schlechten internen Konsistenz (und auch der seiner theoretisch angenommenen Teile: Soziale Einflüsse $\alpha=0.56$, Nähe zum Wohnort $\alpha=0.40$ ) im Folgenden nicht weiter betrachtet. Die untersuchten Studienwahlmotive sind also in der Erwartungskomponente die eigene fachliche Fähigkeitsüberzeugung (abgekürzt als „Fähigkeit“) und in den Wertkomponenten die intrinsischen Werte fachliches Interesse (in den Tabellen als „Fach“ abgekürzt) und berufliches Interesse (abgekürzt als „Beruf“), der gesellschaftliche Wert des Studiums (abgekürzt als „Gesellschaft"), der extrinsische Wert der persönlichen
Nützlichkeit (abgekürzt als „nützlich“) sowie die geringen Kosten durch die (antizipierte) geringe Schwierigkeit des Studiums (abgekürzt als „einfach“).

\section{Ergebnisse zu Forschungsfrage 2}

Die Ergebnisse der Varianzanalysen sind in Tabelle 5 aufgeführt. Bewerberinnen und Bewerber auf ein Lehramtsstudium unterscheiden sich in allen gemessenen Studienwahlmotiven signifikant von den Bewerberinnen und Bewerbern auf andere Studiengänge, wobei das fachliche Interesse niedriger und alle anderen Studienwahlmotive höher als in anderen Studienganggruppen ausgeprägt sind. Diese Unterschiede entsprechen großen Effekten bei beruflichem Interesse (Cohens $d=$ 1.39) und der persönlichen Nützlichkeit $(d=1.07)$, mittleren Effekten in der fachlichen Fähigkeitsüberzeugung $(d=0.56)$, dem gesellschaftlichen Wert des Studiums $(d=0.47)$ und dem fachlichen Interesse $(d=-0.45)$ sowie einem kleinen Effekt bei der antizipierten geringen Schwierigkeit des Studiums $(d=0.30)$. Auch beim diffe- 
Tabelle 5. Ergebnisse der Varianzanalysen

\begin{tabular}{|c|c|c|c|c|c|c|}
\hline & Fähigkeit & Fach & Beruf & Gesellschaft & nützlich & einfach \\
\hline Gruppe/Cluster & $M(S D)$ & $M(S D)$ & $M(S D)$ & $M(S D)$ & $M(S D)$ & $M(S D)$ \\
\hline Lehramt & $4.14^{\mathrm{a}}(0.57)$ & $4.51^{\mathrm{a}}(0.46)$ & $4.86^{a}(0.35)$ & $4.44^{a}(0.56)$ & $3.85^{a}(0.65)$ & $1.98^{a}(0.71)$ \\
\hline Nicht-Lehramt & $3.81^{b}(0.60)$ & $4.69^{b}(0.35)$ & $3.77^{b}(0.95)$ & $4.10^{b}(0.81)$ & $2.99^{b}(0.89)$ & $1.78^{b}(0.65)$ \\
\hline$F(1,688)$ & $49.16^{*}$ & $31.78^{*}$ & $309.14^{\star}$ & $35.40 *$ & $182.85 *$ & $14.37 \star$ \\
\hline Lehramt & $4.14^{\mathrm{a}}(0.57)$ & $4.51^{a}(0.46)$ & $4.86^{a}(0.35)$ & $4.44^{a}(0.56)$ & $3.85^{a}(0.65)$ & $1.98^{a}(0.71)$ \\
\hline Nachhaltigkeit & $3.72^{b}(0.63)$ & $4.77^{b}(0.27)$ & $3.47^{b}(0.92)$ & $4.51^{a}(0.51)$ & $2.66^{b}(0.82)$ & $1.68^{b}(0.60)$ \\
\hline Kultur & $3.83^{b, c}(0.59)$ & $4.70^{b}(0.37)$ & $3.87^{\circ}(0.93)$ & $3.93^{b}(0.86)$ & $3.00^{\circ}(0.84)$ & $1.83^{a, b}(0.70)$ \\
\hline Wirtschaft & $3.96^{\mathrm{a}, \mathrm{c}}(0.54)$ & $4.48^{\mathrm{a}}(0.40)$ & $4.14^{\circ}(0.86)$ & $3.67^{\circ}(0.85)$ & $3.65^{a}(0.78)$ & $1.86^{a, b}(0.62)$ \\
\hline$F(3,686)$ & $19.24^{\star}$ & $20.38^{\star}$ & $124.47^{\star}$ & $45.35 *$ & $96.12^{\star}$ & $6.48 *$ \\
\hline 2.1: Vielseitig & $4.15^{a}(0.53)$ & $4.59^{a}(0.43)$ & $4.68^{\mathrm{a}}(0.47)$ & $4.31^{a}(0.68)$ & $3.75^{\mathrm{a}}(0.66)$ & $2.02^{\mathrm{a}}(0.71)$ \\
\hline 2.2: Fachlich & $3.60^{\mathrm{b}}(0.58)$ & $4.68^{b}(0.36)$ & $3.41^{b}(0.96)$ & $4.09^{b}(0.82)$ & $2.64^{b}(0.81)$ & $1.61^{b}(0.57)$ \\
\hline$F(1,688)$ & 169.66* & $8.91 *$ & $530.58 *$ & $15.71^{\star}$ & $394.13^{*}$ & $63.41^{*}$ \\
\hline 3.1:Vielseitig & $4.27^{a}(0.48)$ & $4.80^{\mathrm{a}}(0.23)$ & $4.68^{a}(0.45)$ & $4.52^{a}(0.50)$ & $3.69^{a}(0.73)$ & $1.89^{a}(0.67)$ \\
\hline 3.2: Fachlich & $3.59^{b}(0.57)$ & $4.78^{a}(0.24)$ & $3.37^{b}(0.99)$ & $4.30^{\mathrm{b}}(0.66)$ & $2.49^{b}(0.79)$ & $1.55^{b}(0.56)$ \\
\hline 3.3: Pragmatisch & $3.75^{\circ}(0.57)$ & $4.10^{\mathrm{b}}(0.39)$ & $4.26^{\circ}(0.84)$ & $3.58^{c}(0.85)$ & $3.64^{\mathrm{a}}(0.63)$ & $2.17^{\circ}(0.69)$ \\
\hline$F(2,687)$ & $117,49 *$ & $391.32 *$ & $193.53 *$ & $117.73 *$ & $196.75^{\star}$ & $44.59 *$ \\
\hline 4.1: Vielseitig Fach & $4.15^{a}(0.50)$ & $4.83^{b}(0.21)$ & $4.65^{\mathrm{a}}(0.42)$ & $4.42^{a, b}(0.54)$ & $3.32^{d}(0.81)$ & $1.43^{d}(0.37)$ \\
\hline 4.2: Fachlich & $3.50^{b}(0.59)$ & $4.77^{b}(0.25)$ & $3.00^{b}(0.88)$ & $4.32^{b}(0.62)$ & $2.49^{b}(0.81)$ & $1.60^{b}(0.55)$ \\
\hline 4.3: Pragmatisch & $3.71^{\circ}(0.57)$ & $4.07^{\circ}(0.40)$ & $4.22^{\circ}(0.87)$ & $3.39^{\circ}(0.84)$ & $3.57^{\circ}(0.71)$ & $1.96^{\circ}(0.56)$ \\
\hline 4.4:Vielseitig Prag & $4.21^{a}(0.52)$ & $4.63^{a}(0.34)$ & $4.58^{a}(0.57)$ & $4.52^{\mathrm{a}}(0.51)$ & $3.88^{\mathrm{a}}(0.68)$ & $2.69^{a}(0.50)$ \\
\hline$F(3,686)$ & $68.21 *$ & $211.61^{\star}$ & $217.41^{\star}$ & $102.21 *$ & $94.73^{*}$ & $231,10 *$ \\
\hline
\end{tabular}

Anmerkung: *Die Haupteffekte der Varianzanalysen sind alle signifikant mit mit $p \leq 0.003$. a, b, c, d: Die Mittelwertunterschiede der Studienwahlmotive sind in den jeweiligen Gruppen für unterschiedliche Buchstaben signifikant (Post-hoc-Tests mit Bonferroni-Korrektur; $p<0.05$ ).

Tabelle 6. Werte zu den K-Means-Clusteranalysen

\begin{tabular}{llllr}
\hline$K$ & $S Q_{\text {in }}(K)$ & $\eta^{2}{ }_{K}$ & $P R E_{K}$ & \multicolumn{1}{c}{$F_{\text {max }}$} \\
\hline 1 & 4134.00 & .00 & - & 0.00 \\
2 & 3364.43 & .19 & .19 & 157.37 \\
3 & 2837.22 & .31 & .16 & 157.00 \\
4 & 2547.77 & .38 & .10 & 142.37 \\
5 & 2352.99 & .43 & .08 & 129.62 \\
6 & 2175.63 & .47 & .08 & 123.14 \\
\hline
\end{tabular}

Anmerkung: Streuungsquadratsumme in den Clustern $S Q_{\text {in }}(K)$, erklärte Streuung $\eta^{2}$, proportionale Fehlerverbesserung gegenüber einer vorausgehenden Clusterlösung $P R E_{K}$ und maximale $F_{\text {maxk }} F$-Statistik für die verschiedenen Clusteranzahlen $K$.

renzierteren Vergleich der vier Studienganggruppen zeigen sich überwiegend signifikante Unterschiede, wobei Ähnlichkeiten am häufigsten zwischen Bewerberinnen und Bewerbern auf ein Lehramtsstudium und Bewerberinnen und Bewerbern auf ein wirtschaftswissenschaftliches Studium zu finden sind. Zudem gehören Bewerberinnen und Bewerber auf ein Lehramtsstudium jeweils zu der Gruppe, in der die verschiedenen Studienwahlmotive - mit Ausnahme des Fachinteresses - am höchsten ausgeprägt sind.

\section{Ergebnisse zu Forschungsfrage 3 und 4}

Die Ergebnisse zur Bewertung der $K$-Means Clusteranalysen für die festgesetzten Clusteranzahlen $K=1, \ldots, 6$ sind in Tabelle 6 aufgeführt. Mit Blick auf die erklärte Streuung $\eta_{K}^{2}$ kommen die 2-, 3- und 4-Clusterlösung in Betracht. Bezogen auf die proportionale Fehlerverbesserung im Vergleich zur Vorgängerlösung $P R E_{K}$ und die $F_{\operatorname{maxK}}-$ Statistik hat die 2-Clusterlösung die besten Werte. Die 3-Clusterlösung weist ähnlich gute Werte auf. 


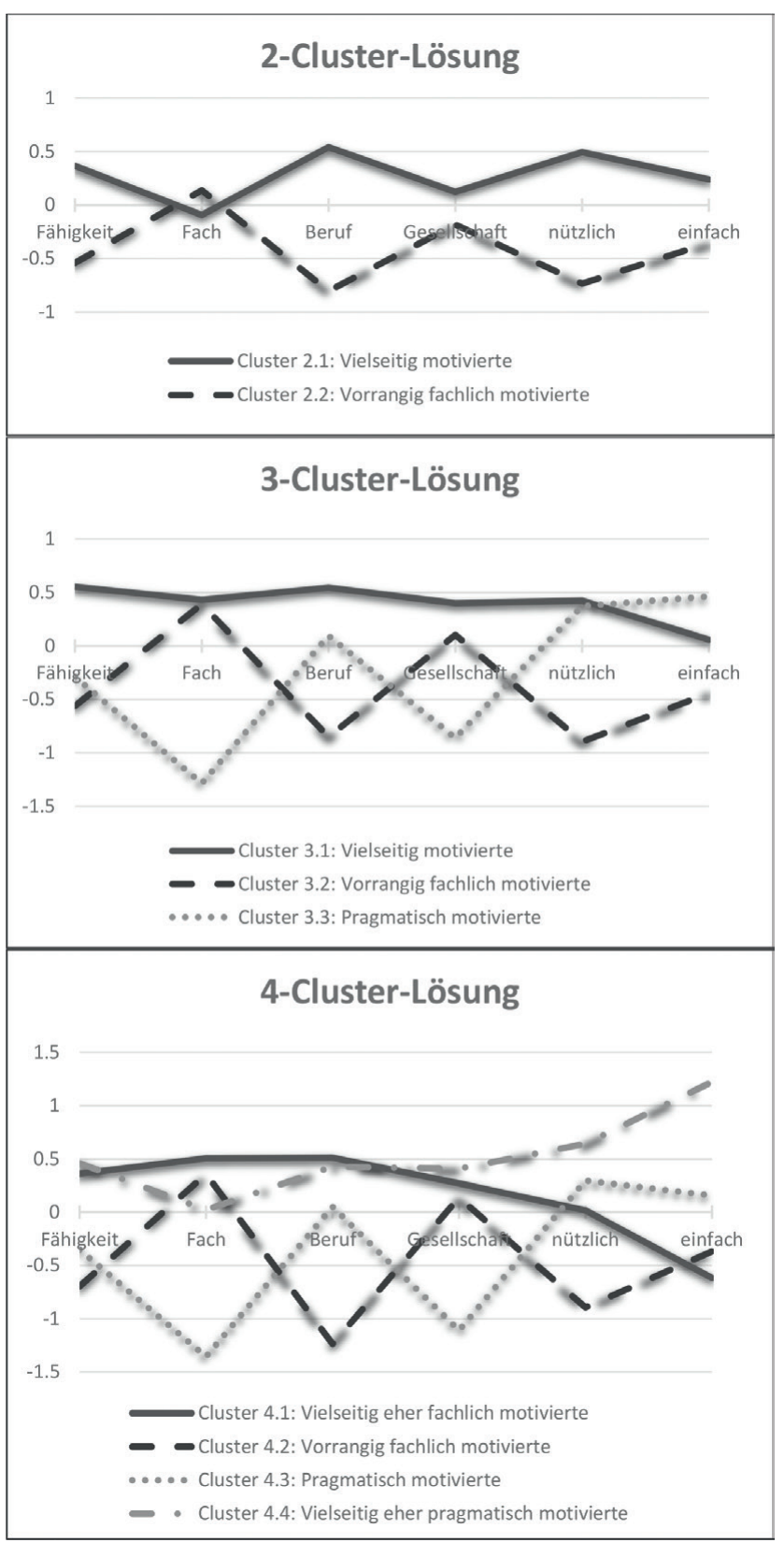

Abbildung 2. z-standardisierte Mittelwerte der jeweiligen Cluster-Lösungen.

Für eine inhaltliche Betrachtung der verschiedenen Lösungen werden im Folgenden die jeweiligen Cluster der 2-, 3- und 4-Clusterlösung von der 3-Clusterlösung ausgehend beschrieben. Dabei werden mit Blick auf Forschungsfrage 4 auch die in den Clustern vertretenen Studiengänge betrachtet. Deskriptive Werte zu diesen Lösungen finden sich in Tabelle 5 . Ein Liniendiagramm zu den $z$-standardisierten Mittelwerten der einzelnen Cluster ist in Abbildung 2 gegeben. Eine Visualisierung der Veränderung der Zuordnungen für die 2-, 3- und 4-Clusterlösung, unter Berücksichtigung der verschiedenen Studiengänge, findet sich in Abbildung 3.

\section{Cluster 3.1: Die vielseitig motivierten}

Charakteristisch für Bewerberinnen und Bewerber aus diesem Cluster ist eine im Vergleich mit den anderen Gruppen hoch ausgeprägte Zustimmung zu allen - oder dem Großteil der - erfassten Studienwahlmotive. Ausschlaggebend für die Studienwahl von Personen aus diesem Cluster sind nicht einzelne Motive, sondern das $\mathrm{Zu}-$ sammenspiel von fachlichen und beruflichen Interessen, gesellschaftlichen und persönlichen Nützlichkeitswerten sowie fachlichen Fähigkeitsüberzeugungen.

Dieses Muster ist deutlich in der 2- und 3-Clusterlösung wiederzufinden. In der 4-Clusterlösung wird es in zwei Cluster unterteilt, die jeweils entweder die fachlichen Interessen (Cluster 4.1: Vielseitig eher fachlich motivierte) oder die persönliche Nützlichkeit und Kosten (Cluster 4.4: Vielseitig eher pragmatisch motivierte) stärker gewichten.

In der 2-Clusterlösung werden $96 \%$ der Bewerberinnen und Bewerber auf ein Lehramtsstudium diesem Cluster zugeordnet, in der 3-Clusterlösung sind es noch $69 \%$. Bewerberinnen und Bewerber auf ein Lehramtsstudium stellen auch einen Großteil der Personen in diesem Cluster. In der 2-Clusterlösung sind 59\% der vielseitig motivierten Bewerberinnen und Bewerber auf ein Lehramtsstudium, in der 3-Clusterlösung sind es $57 \%$ (siehe Tabelle 7 für weitere Werte).

\section{Cluster 3.2: Die vorrangig fachlich motivierten}

Bewerberinnen und Bewerber aus diesem Cluster wählen ihr Studium vorrangig aufgrund fachlicher Interessen. Auch gesellschaftliche Werte sind hoch ausgeprägt (allerdings etwas geringer als bei den vielseitig motivierten), wohingegen berufsbezogene Interessen und die persönliche Nützlichkeit im Vergleich zu Bewerberinnen und Bewerbern aus anderen Clustern eine deutlich untergeordnete Bedeutung für die Studienwahl haben.

Dieses Muster findet sich auch in der 2- und 4-Clusterlösung wieder.

In der 2-Clusterlösung werden nur $4 \%$ der Bewerberinnen und Bewerber auf ein Lehramtsstudium diesem Cluster zugeordnet, in der 3-Clusterlösung sind es unter $3 \%$, in der 4-Clusterlösung unter 1\%. Der Großteil der Personen in diesem Cluster sind Bewerberinnen und Bewerber auf einen Studiengang aus den Studienganggruppen Nachhaltigkeit oder Kultur. Über $92 \%$ der Bewerberinnen und Bewerber, die diesem Cluster in der 3-Clusterlösung zugeordnet sind, haben sich für einen Studiengang aus den Studienganggruppen Nachhaltigkeit oder Kultur entschieden, in der 2-Clusterlösung sind es $87 \%$, in der 4-Clusterlösung sogar $96 \%$ (siehe Tabelle 7 für weitere Werte).

\section{Cluster 3.3: Die pragmatisch motivierten}

Bewerberinnen und Bewerber aus diesem Cluster wählen ihr Studium vergleichsweise stärker aufgrund prag- 


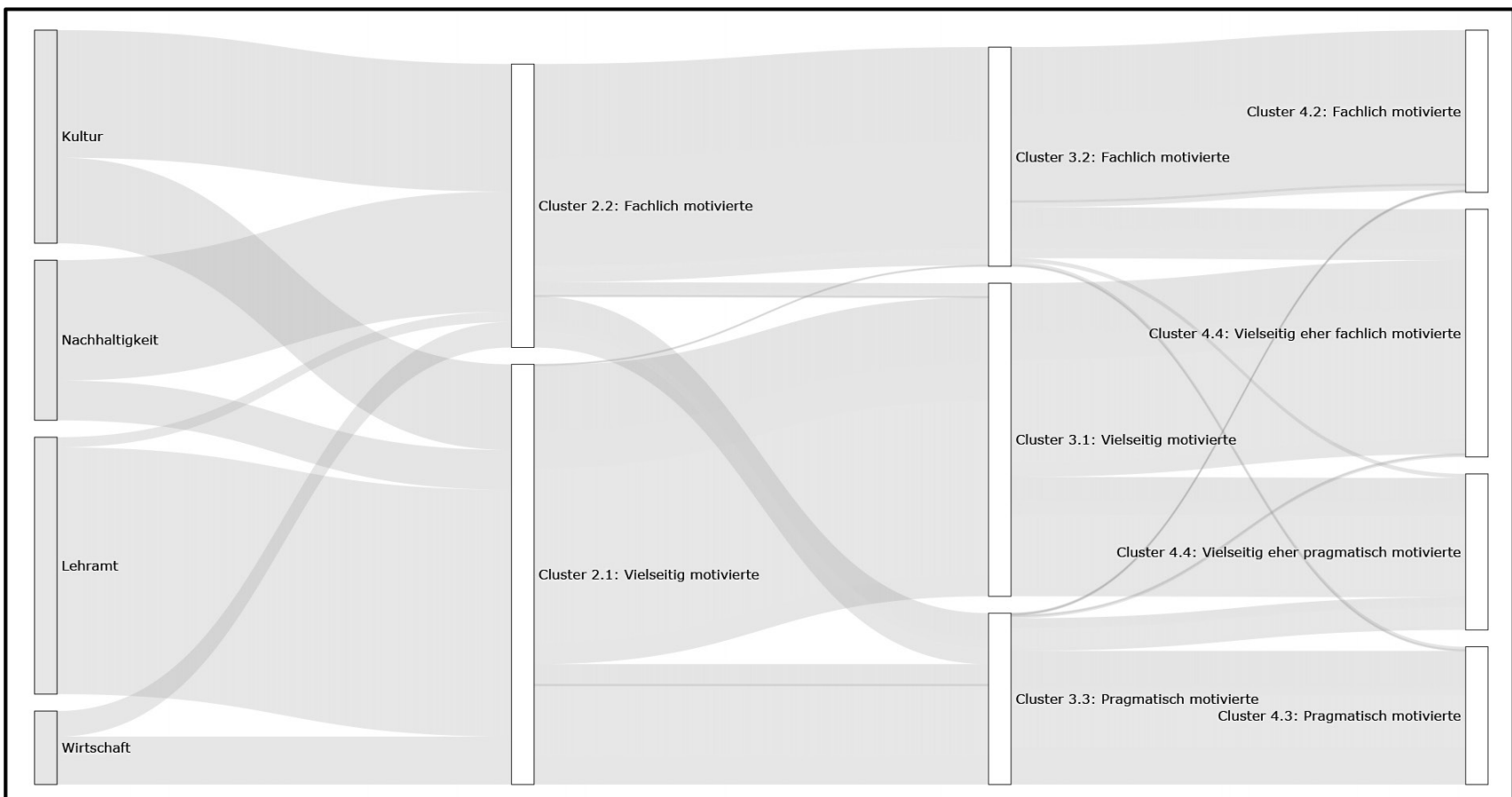

Abbildung 3. Verteilung der vier Studienganggruppen auf die verschiedenen Cluster. Die Höhe der Balken entspricht der Anzahl der Bewerberinnen und Bewerber in den jeweiligen Clustern, die verschiedenen Farben (siehe ESM 1) den verschiedenen Studienganggruppen.

matischer Überlegungen, z.B. hinsichtlich finanzieller Sicherheit, Vereinbarkeit von Familie und Beruf (persönliche Nützlichkeit) und einer (erwarteten) geringeren Schwierigkeit des gewählten Studiengangs (Kosten). Charakteristisch für dieses Cluster ist zudem, dass fachliche und gesellschaftliche Motive deutlich weniger ausgeprägt sind als bei Bewerberinnen und Bewerbern aus anderen Clustern.

In der 2-Clusterlösung ist dieses Cluster noch nicht erkennbar. Das in der 3-Clusterlösung gefundene Muster tritt auch in der 4-Clusterlösung wieder auf. In der 4-Clusterlösung ergibt sich zudem ein ähnliches Cluster auf einem insgesamt höheren Niveau (Cluster 4.4: Vielseitig eher pragmatisch motivierte), das zur weiteren Ausdifferenzierung der vielseitig motivierten beiträgt.

In der 3-Clusterlösung werden etwa $29 \%$ der Bewerberinnen und Bewerber auf ein Lehramtsstudium diesem Cluster zugeordnet, in der 4-Clusterlösung sind es ca. $21 \%$ (knapp $37 \%$ werden den vielseitig eher pragmatisch motivierten zugeordnet). Bewerberinnen und Bewerber auf einen Studiengang zur Nachhaltigkeit sind unter den pragmatisch motivierten kaum vertreten und stellen weniger als $6 \%$ der Personen in diesem Cluster. Bewerberinnen und Bewerber auf ein wirtschaftswissenschaftliches Studium stellen, trotz ihrer zahlenmäßigen Unterrepräsentation in dieser Stichprobe, jeweils ca. ein Viertel der pragmatisch motivierten, sowohl in der 3- als auch der 4-Clusterlösung (siehe Tabelle 7 für weitere Werte).

\section{Diskussion}

\section{Zusammenfassung und Diskussion der Ergebnisse zu Forschungsfrage 1}

Für die vorliegende Studie wurden Studienwahlmotive auf der Grundlage des Erwartungs-Wert-Modells durch Adaption vorhandener, für Lehramtsstudentinnen und -studenten entwickelter Instrumente - insbesondere des „Fragebogens zur Erfassung der Motivation für die Wahl des Lehramtsstudiums“" (FEMOLA, Pohlmann \& Möller, 2010) -, mithilfe standardisierter und reliabler Fragebogenskalen studiengangübergreifend (d.h. auch von Bewerberinnen und Bewerbern auf Nicht-Lehramtsstudiengänge) erfasst. Dabei konnte die theoretisch angenommene Faktorenstruktur des so entwickelten Fragebogens sowohl mithilfe einer explorativen als auch einer konfirmatorischen Faktoranalyse bestätigt werden. Bemerkenswert ist an dieser Stelle, dass die Modell-Fit-Werte der konfirmatorischen Faktorenanalyse in dieser Studie sogar besser ausfallen als in der ursprünglichen (Pohlmann \& Möller, 2010) und weiteren (Retelsdorf \& Möller, 2012; Rutsch et al., 2020) Stu- 
Tabelle 7. Anzahlen der Bewerberinnen und Bewerber aus den verschiedenen Studienganggruppen in den jeweiligen Clustern

\begin{tabular}{|c|c|c|c|c|c|}
\hline Cluster & Lehramt & Nachhaltigkeit & Kultur & Wirtschaft & Gesamt \\
\hline 2.1:Vielseitig & $242(96.0 \%)$ & $39(24.8 \%)$ & $84(40.2 \%)$ & $47(65.3 \%)$ & $412(59.7 \%)$ \\
\hline 2.2: Fachlich & $10(4.0 \%)$ & $118(75.2 \%)$ & $125(59.8 \%)$ & $2534.7 \%)$ & $278(40.3 \%)$ \\
\hline 3.1:Vielseitig & $174(69.0 \%)$ & $40(25.5 \%)$ & $72(34.4 \%)$ & $21(29.2 \%)$ & $307(44.5 \%)$ \\
\hline 3.2: Fachlich & $6(2.4 \%)$ & 107 (68.2\%) & $92(44.0 \%)$ & $10(13.9 \%)$ & $215(31.2 \%)$ \\
\hline 3.3: Pragmatisch & $72(28.6 \%)$ & $10(6.4 \%)$ & $45(21.5 \%)$ & $41(56.9 \%)$ & $168(24.3 \%)$ \\
\hline 4.1: Vielseitig Fach & $107(42.5 \%)$ & $46(29.3 \%)$ & $69(33.0 \%)$ & $21(29.2 \%)$ & $243(35.2 \%)$ \\
\hline 4.2: Fachlich & $1(0.4 \%)$ & $86(54.8 \%)$ & $67(32.1 \%)$ & $5(6.9 \%)$ & $159(23.0 \%)$ \\
\hline 4.3: Pragmatisch & $52(20.6 \%)$ & $8(5.1 \%)$ & $39(18.7 \%)$ & $36(50.0 \%)$ & $135(19.6 \%)$ \\
\hline 4.4:Vielseitig Prag & $92(36.5 \%)$ & $17(10.8 \%)$ & $34(16.3 \%)$ & 10 (13.9\%) & $153(22.2 \%)$ \\
\hline
\end{tabular}

Anmerkung: In Klammern der prozentuale Anteil der Bewerberinnen und Bewerber aus den jeweiligen Studienganggruppen in den Clustern.

dien zur Überprüfung der Faktorenstruktur des FEMOLA. Die Reliabilitäten der so erhaltenen Skalen sind mit einer Ausnahme im akzeptablen bis sehr guten Bereich. Wahrscheinlich hätten durch einen weniger „sparsamen Einsatz" von Items zu sozialen Einflüssen aus dem FEMOLA (vgl. Pohlmann \& Möller, 2010) auch hier bessere Werte erreicht werden können. Insgesamt konnte die auf der Basis des Erwartungs-Wert-Modells für Lehramtsstudierende entwickelte Klassifikation von Studienwahlmotiven auf andere Studiengänge übertragen und reliabel erfassbar gemacht werden, was Forschungsfrage 1 positiv beantwortet.

\section{Zusammenfassung und Diskussion der Ergebnisse zu Forschungsfrage 2}

Studiengangübergreifend ist das Fachinteresse das am höchsten ausgeprägte Studienwahlmotiv, gefolgt von gesellschaftlichen Werten, dem Interesse an einem bestimmten Beruf und der eigenen Fähigkeitsüberzeugung bezogen auf die gewählten Studienfächer. Diese Reihenfolge zeigt sich im Wesentlichen auch in den im Theorieteil vorgestellten Ergebnisse zu Studienwahlmotiven von Lehramtsstudentinnen und -studenten im deutschsprachigen Raum (Biermann et al., 2019; Dörrenbächer-Ulrich et al., 2019; König et al., 2013, 2018; Micknass et al., 2019; Pohlmann \& Möller, 2010; Retelsdorf \& Möller, 2012) und ist offenbar ein Muster, das nicht nur für das Lehramt gilt (was mit Blick auf Studiengänge, die keineswegs auf ,den einen Beruf" hinauslaufen, durchaus überraschen mag). Das in Studien zum Lehramtsstudium in der Regel nicht explizit erhobene Interesse an einem bestimmten Beruf ist bei den hier befragten Bewerberinnen und Bewerbern auf ein Lehramtsstudium sogar höher ausgeprägt als das Fachinteresse. Dies unterstreicht die enge Verzahnung von Studien- und Berufswahlmotiven für das Lehramtsstudium, das wie kaum ein anderer Studiengang auf einen bestimmten Beruf ausgerichtet ist (Spinath et al., 2005). Tatsächlich ist das Interesse an einem bestimmten Beruf auch das Studienwahlmotiv, in dem sich Bewerberinnen und Bewerber auf ein Lehramtsstudium am deutlichsten von Bewerberinnen und Bewerbern auf andere Studiengänge unterscheiden (vgl. Tabelle 5).

Bis auf die (antizipierte) geringe Schwierigkeit des Studiums als Studienwahlmotiv, das studiengangübergreifend wenig Zustimmung findet, unterscheiden sich die Bewerberinnen und Bewerber auf ein Lehramtsstudium in ihren Studienwahlmotiven von jeweils mindestens zwei der drei anderen Studienganggruppen signifikant. Das Fachinteresse ist bei den Bewerberinnen und Bewerbern auf ein Lehramtsstudium und auf die wirtschaftswissenschaftlichen Studiengänge vergleichsweise niedriger. Alle anderen Studienwahlmotive sind bei den Bewerberinnen und Bewerbern auf ein Lehramtsstudium am höchsten ausgeprägt. Die Studienwahlmotive von Bewerberinnen und Bewerbern auf ein Lehramtsstudium unterscheiden sich also in vielerlei Hinsicht von denen der anderen Studienganggruppen, was Frage 2 positiv beantwortet. Die auch absolut gesehen hohen Ausprägungen in fast allen erfassten Studienwahlmotiven bei den Bewerberinnen und Bewerbern auf ein Lehramtsstudium unterstützt insbesondere für diese die eingangs vorgestellte These, dass Studienwahlentscheidungen nicht nur aufgrund einzelner Motive, sondern vielmehr durch eine Abwägung verschiedener Motive, getroffen werden.

\section{Zusammenfassung und Diskussion der Ergebnisse zu Forschungsfrage 3 und 4}

Diese Interpretation wird auch durch die Betrachtung der Clusterlösungen unterstützt: Ein Großteil der Bewerberinnen und Bewerber auf ein Lehramtsstudium wird den vielseitig motivierten Profilen zugeordnet. Dabei identifiziert 
insbesondere die 3-Clusterlösung inhaltlich gut zu interpretierende Motivprofile, die auch durch die Mitbetrachtung der 2- und 4-Clusterlösung und insbesondere der in Abbildung 3 dargestellten Zusammensetzung aus den Studienganggruppen besser verstanden werden kann: Auf den ersten Blick scheint die 3-Clusterlösung die in der Literatur identifizierte Unterteilung in ein vorrangig intrinsisch motiviertes Profil (hier: die vorrangig fachlich motivierten), ein vorrangig extrinsisch motiviertes Profil (hier: die pragmatisch motivierten), und ein ausgewogen (hoch) motiviertes Profil (hier: die vielseitig motivierten) zu replizieren (z.B. Biermann et al., 2019; Billich-Knapp et al., 2012; König et al., 2018). Bemerkenswert ist dabei allerdings, dass Bewerberinnen und Bewerber auf ein Lehramtsstudium kaum in Cluster 3.1 der vorrangig fachlich motivierten vertreten sind. Die in der Literatur identifizierten drei Profile entsprechen wohl eher den drei Clustern, auf die sich die Bewerberinnen und Bewerber auf ein Lehramtsstudium in der 4-Clusterlösung verteilen, wobei das vorrangig fachlich motivierte Profil ein hier neu identifiziertes Profil ist, das für Lehramtsstudiengänge nicht existiert.

Lehramtsstudentinnen und -studenten mit einer hohen extrinsischen und vergleichsweise niedrigen intrinsischen Motivation werden gelegentlich als „Risikogruppe“ (Biermann et al., 2019; Boeger, 2016; Rothland, 2013) betrachtet, die mit Blick auf Studienstrategienutzung und Studienzufriedenheit (Künsting \& Lipowsky, 2011; Wach et al., 2016) sowie Burnout-Symptome (McLean et al., 2019; Reichl et al., 2014) ungünstigere Voraussetzungen haben. In der vorliegenden Studie entsprechen diese vor allem der Gruppe der pragmatisch motivierten. Diese sind in der Studienganggruppe "Nachhaltigkeit" kaum vertreten, machen in der Studiengruppe „Kultur“ ca. 20\%, im ,Lehramt“ $20-30 \%$ und in der Studiengruppe „Wirtschaft" sogar über $50 \%$ aus (siehe Tabelle 7). Eine empirische Untersuchung, inwieweit sich die für Lehramtsstudentinnen und -studenten gefundenen Risikomerkmale auch auf andere Studiengänge (hier insbesondere: Studiengänge des Bereichs „Wirtschaft") übertragen lassen, stellt einen unmittelbar zu verfolgenden, nächsten Forschungsschritt dar.

\section{Implikationen für die Lehrerinnen- und Lehrerbildung}

Die in dieser Studie vorgenommenen studiengangübergreifenden Vergleiche von Studienwahlmotiven tragen sowohl in der variablenzentrierten (Forschungsfrage 2) als auch in der personenzentrierten profilanalytischen (Forschungsfragen 3 und 4) Betrachtung zu einem besseren Verständnis von Studienwahlmotivationen bei, was auch für die praktische Ausgestaltung der Lehrerinnen- und Lehrerbildung relevant ist. So erweisen sich Bewerberin- nen und Bewerber auf ein Lehramtsstudium absolut und auch relativ gesehen als überwiegend vielfältig hochmotiviert. Im Vergleich mit anderen Studiengängen wird allerdings deutlich, dass für Bewerberinnen und Bewerber auf ein Lehramtsstudium das fachliche Interesse (sowohl im personenübergreifenden Vergleich als auch im intrapersonellen Vergleich der verschiedenen Motive) eine geringere Bedeutung hat als für Bewerberinnen und Bewerber auf andere Studiengänge. Mit Blick auf die klare Berufsausrichtung eines Lehramtsstudiums und die Vielfalt professioneller Kompetenzen und Aufgaben von Lehrkräften (siehe z.B. Krauss et al., 2020) können derart vielfältige Motive für ein Lehramtsstudium durchaus als angemessen und erwartbar angesehen werden. Dabei ist zu beachten, dass die berufliche Orientierung auch für das fachliche Interesse ein große Rolle spielt: Lehramtsstudierende im Fach Mathematik sind z.B. stärker an schulmathematischen Inhalten, Ein-Fach-Studierende stärker an hochschulmathematischen Inhalten (bzw. allgemein stärker fachlich) interessiert (Ufer et al., 2017). Diese hohe Bedeutung der beruflichen Interessen von Lehramtsstudentinnen und -studenten kann dazu führen, dass bei einer (zu) starken Fokussierung auf abstrakte fachliche Inhalte, durch die ebendiese Relevanz für den späteren Beruf nicht deutlich wird, Motivationsschwierigkeiten und Frustrationen auftreten (Göller, 2020; Liebendörfer, 2018). Dies stärkt die Notwendigkeit hochschuldidaktischer Entwicklungsprojekte, die versuchen, diese Berufsrelevanz explizit im Studium zu verdeutlichen, relevantes schulbezogenes Fachwissen für Studierende nachvollziehbar zu identifizieren oder durch einen stärkeren Praxisbezug die Motivation von Lehramtsstudentinnen und -studenten gezielt zu unterstützen (z. B. Rach, 2019 für einen Überblick). Bei dieser motivationszentrierten Sichtweise sollte allerdings nicht vergessen werden, dass insbesondere das Fachwissen als notwendige Voraussetzung für die Entwicklung fachdidaktischen Wissens gilt, welches wiederum zentral für die Qualität schulischer Lehr-Lern-Prozesse ist (Kleickmann et al., 2017). Insgesamt zeigt sich die Bedeutung von universitären Lerngelegenheiten zur Vernetzung verschiedener Wissensbereiche, sowohl aus motivationaler Sicht als auch mit Blick auf den Aufbau professioneller Kompetenzen (Kleickmann \& Hardy, 2019).

Diese Beispiele verdeutlichen, dass eine vergleichsweise geringere fachliche Motivation von Lehramtsstudentinnen und -studenten vielfach berichtet und mittlerweile auch durch praktische Maßnahmen adressiert wird. Diese Sichtweise wird durch die variablenzentrierten Studiengangvergleiche (Forschungsfrage 2) unterstützt und liefert ein für die Lehramtsausbildung kritisches Bild, da zu erwarten ist, dass sich ein geringeres fachliches Interesse ungünstig auf die zukünftige Unterrichtsgestaltung auswirken kann. Tatsächlich wird die Motivation von Lehrkräften als Teil ihrer 
professionellen Kompetenz betrachtet, bei der sich insbesondere der Enthusiasmus für das Unterrichten als prädiktiv für die Qualität von Unterricht erweist (Kunter, 2011). Welche der hier untersuchten Studienwahlmotive einen solchen Enthusiasmus vorhersagen müsste allerdings erst noch untersucht werden. Der personenzentrierte Ansatz mithilfe der Motivprofile liefert hier zudem ein differenzierteres Bild: Die 3- und 4-Clusterlösung legen nahe, dass diese Mittelwertunterschiede vor allem durch den relativ hohen Anteil an pragmatisch motivierten Bewerberinnen und Bewerbern auf ein Lehramtsstudium zu begründen sind (in den Wirtschaftswissenschaften ist dieser Anteil sogar noch um einiges höher). Maßnahmen zur Unterstützung dieser „Risikogruppe" sind sicherlich angebracht und wichtig. Allerdings muss auch berücksichtigt werden, dass keineswegs alle Bewerberinnen und Bewerber auf ein Lehramtsstudium zu dieser „Risikogruppe“ gehören und dass der Großteil dieser mit Blick auf die Studienwahlmotive, insbesondere dem fachlichen Interesse, sicherlich keine Negativselektion darstellt (vgl. auch Neugebauer, 2013; Roloff Henoch et al., 2015; Spinath et al., 2005): In der 3-Clusterlösung gehören $69 \%$ der Bewerberinnen und Bewerber auf ein Lehramtsstudium zur Gruppe der vielseitig motivierten. In der 4-Clusterlösung gehört fast die Hälfte der Bewerberinnen und Bewerber auf ein Lehramtsstudium zur Gruppe der vielseitig eher fachlich motivierten. Dies sind jeweils die Gruppen, in denen das fachliche Interesse am höchsten ausgeprägt ist (auf einem Niveau mit den fachlich motivierten). Insgesamt legt vor allem die Betrachtung der Clusterlösungen nahe, dass eine pauschale Beurteilung von Lehramtsstudierenden aufgrund von Mittelwertvergleichen mit anderen Studienganggruppen (z.B. bzgl. des Fachinteresses oder auch bzgl. anderer Studienwahlmotive) der Heterogenität der Bewerberinnen und Bewerber auf ein Lehramtsstudium nicht gerecht wird. Vielmehr zeigt sich ein großer Teil von Bewerberinnen und Bewerbern auf ein Lehramtsstudium als auch fachlich hoch motiviert. Allerdings existiert leider ebenso eine ( $\mathrm{zu}$ ) große Anzahl an eher pragmatisch motivierten Bewerberinnen und Bewerbern, was mit Blick auf oben genannte Risikomerkmale und auch die zukünftige Unterrichtsgestaltung kritisch zu diskutieren ist.

\section{Limitationen}

Eine wesentliche Limitation mit Blick auf die Verallgemeinerbarkeit der hier vorgestellten Ergebnisse ergibt sich aus der Zusammensetzung der Stichprobe, die nicht repräsentativ für Bewerberinnen und Bewerber an deutschen, geschweige denn internationalen, Universitäten ist. Die hier befragten Bewerberinnen und Bewerber haben sich alle an einer bestimmten Universität beworben, wodurch aufgrund der örtlichen Nähe Bewerberinnen und Bewerber aus bestimmten Bundesländern überrepräsentiert sind. Außerdem unterscheiden sich Universitäten zum Teil deutlich in den von ihnen angebotenen Studiengängen. Die Beschreibung der Stichprobe im Methodenteil zeigt, dass einige Studiengänge, die an anderen Universitäten stark vertreten sind, hier nicht existent sind. Mit Blick auf das Lehramtsstudium sind diesbezüglich insbesondere die gymnasialen Lehramtsstudiengänge zu nennen. Dennoch liefert die Studie erste Einblicke in echte studiengangübergreifende Vergleiche von Studienwahlmotiven und verortet die oftmals allein absoluten Werte von Bewerberinnen und Bewerbern auf ein Lehramtsstudium hier im Kontext von drei weiteren Studienganggruppen (Nachhaltigkeit, Kultur, Wirtschaft). Damit liefert die Studie neue Ansätze und interessante Ergebnisse zum besseren Verstehen (nicht nur) lehramtsspezifischer Studienwahlmotive.

Aber auch für die Bewerberinnen und Bewerber an der Leuphana Universität Lüneburg ist die hier untersuchte Stichprobe nicht repräsentativ. Insgesamt sind die Studienteilnehmerinnen und -teilnehmer bzgl. ihrer mittleren Abiturnote und ihres Geschlechterverhältnisses zur Gesamtheit der Teilnehmerinnen und Teilnehmer am Auswahlverfahren und auch der später Immatrikulierten zwar ähnlich, allerdings sind z.B. Bewerberinnen und Bewerber auf ein Grund-Haupt-Realschul-Lehramtsstudium vergleichsweise überrepräsentiert und Bewerberinnen und Bewerber auf die Studienganggruppe Wirtschaft unterrepräsentiert. Daraus resultiert auch, dass in der Gesamtstichprobe Bewerberinnen etwas überrepräsentiert sind.

Schließlich muss berücksichtigt werden, dass ganz allgemein bei Selbstberichten Antworten möglicherweise aufgrund sozialer Erwünschtheit oder Generalisierungsproblemen positiv verzerrt sind. Dies betrifft die hier vorgestellte Studie eventuell besonders, da Bewerberinnen und Bewerber durch die Gesamtsituation an den Auswahltagen (trotz einer Betonung der anonymisierten Datenverarbeitung) möglicherweise "geframed“ sind sich besonders positiv darzustellen. Andererseits ermöglicht das Setting dieser Studie, diese Problematik bewusst zu reduzieren, da diese Situation letztlich für alle Befragten gilt. Gerade der Vergleich unterschiedlicher Studienganggruppen ermöglicht so mindestens einen belastbaren relativen Vergleich von Studiengangmotiven - unabhängig von der Thematik sozialer Erwünschtheit.

\section{Elektronisches Supplement (ESM)}

Das elektronische Supplement ist mit der Online-Version dieses Artikels verfügbar unter https://doi.org/10.1024/ 1010-0652/a000317

ESM 1. Abbildung 3 in Farbe (PDF) 


\section{Literatur}

Afrianto, A. (2014). „Because teaching is like a plantation of dakwah": Understanding complexities in choosing to be a teacher in Indonesia. Australian Journal of Educational and Developmental Psychology, 14, 51-59.

Azman, N. (2013). Choosing teaching as a career: Perspectives of male and female Malaysian student teachers in training. European Journal of Teacher Education, 36(1), 113-130. https://doi. org/10.1080/02619768.2012.678483

Bacher, J., Pöge, A. \& Wenzig, K. (2010). Clusteranalyse: Anwendungsorientierte Einführung in Klassifikationsverfahren (3., erg. vollst. überarb. und neu gestaltete Aufl). Oldenbourg.

Baumert, J. \& Kunter, M. (2006). Stichwort: Professionelle Kompetenz von Lehrkräften. Zeitschrift für Erziehungswissenschaft, 9(4), 469 - 520. https://doi.org/10.1007/s11618-006-0165-2

Becker, F. (2019). Motivation und Motive: Definitionen und Eigenschaften. In F. Becker, Mitarbeiter wirksam motivieren (S.19-25). Springer Berlin Heidelberg. https://doi.org/10.1007 1978-3-662-57838-4_3

Biermann, A., Dörrenbächer-Ulrich, L., Grassmé, I., Perels, F., GläserZikuda, M. \& Brünken, R. (2019). Hoch motiviert, engagiert und kompetent: Eine profilanalytische Untersuchung zur Studienund Berufswahlmotivation von Lehramtsstudierenden: Nutzung von Lerngelegenheiten und Kompetenzen im Praktikum. Zeitschrift für Pädagogische Psychologie, 1-13. https://doi.org/ 10.1024/1010-0652/a000242

Billich-Knapp, M., Künsting, J. \& Lipowsky, F. (2012). Profile der Studienwahlmotivation bei Grundschullehramtsstudierenden. Zeitschrift für Pädagogik, 58(5), 696 - 719.

Boeger, A. (2016). Risikomerkmale und Erfolgsprädiktoren des Lehramtsstudiums: Ergebnisse einer Erstsemesterbefragung. In A. Boeger (Hrsg.), Eignung für den Lehrerberuf (S.59-90). Springer Fachmedien Wiesbaden. https://doi.org/10.1007/9783-658-10041-4_4

Brookhart, S. M. \& Freeman, D. J. (1992). Characteristics of Entering Teacher Candidates. Review of Educational Research, 62(1), 37-60.

Dörrenbächer-Ulrich, L., Biermann, A., Brünken, R. \& Perels, F. (2019). Studienwahlmotivation von Lehramtsstudierenden und Aspekte ihrer professionellen Kompetenz: Eine profilanalytische Untersuchung. Zeitschrift für Entwicklungspsychologie und Pädagogische Psychologie, 51(1), 45-61. https://doi.org/1 0.1026/0049-8637/a000208

Eccles, J. S. \& Wigfield, A. (2002). Motivational Beliefs, Values, and Goals. Annual Review of Psychology, 53(1), 109 - 132. https://doi. org/10.1146/annurev.psych.53.100901.135153

Fischer, B., Paul, A. \& Bisterfeld, M. (2019). Warum entscheiden sich Studierende für ein Lehramtsstudium?: Ein Vergleich zwischen Sport- und Mathematikstudierenden. German Journal of Exercise and Sport Research, 49(1), 56-64. https://doi.org/ 10.1007/s12662-018-0556-6

Fray, L. \& Gore, J. (2018). Why people choose teaching: A scoping review of empirical studies, 2007 -2016. Teaching and Teacher Education, 75,153 - 163. https://doi.org/10.1016/j.tate.2018.06.009

Goller, M., Ursin, J., Vähäsantanen, K., Festner, D. \& Harteis, C. (2019). Finnish and German student teachers' motivations for choosing teaching as a career. The first application of the FITChoice scale in Finland. Teaching and Teacher Education, 85, 235 - 248. https://doi.org/10.1016/j.tate.2019.06.023

Göller, R. (2020). Selbstreguliertes Lernen im Mathematikstudium. Springer Fachmedien Wiesbaden. https://doi.org/10.1007/9783-658-28681-1

Hanfstingl, B. \& Mayr, J. (2007). Prognose der Bewährung im Lehrerstudium und im Lehrerberuf. Journal für Lehrerinnen- und Lehrerbildung, 7, 48-56.
Heckhausen, J. \& Heckhausen, H. (Hrsg.). (2018). Motivation und Handeln. Springer Berlin Heidelberg. https://doi.org/10.1007/ 978-3-662-53927-9

Heublein, U., Ebert, J., Hutzsch, C., Isleib, S., König, R., Richter, J. \& Woisch, A. (2017). Zwischen Studienerwartungen und Studienwirklichkeit. Deutsches Zentrum für Hochschul- und Wissenschaftsforschung GmbH.

Kiel, E., Heimlich, U., Markowetz, R. \& Weiß, S. (2015). Gemeinsam und doch unterschiedlich - Ein Vergleich der Berufswahlmotive von Studierenden der verschiedenen sonderpädagogischen Fachrichtungen und der Regelschularten. Empirische Sonderpädagogik, 7, 300-319.

Kılınç, A., Watt, H. M. G. \& Richardson, P. W. (2012). Factors Influencing Teaching Choice in Turkey. Asia-Pacific Journal of Teacher Education, 40(3), 199 -226. https://doi.org/10.1080/135986 6X.2012.700048

Kleickmann, T. \& Hardy, I. (2019). Vernetzung professionellen Wissens angehender Lehrkräfte im Lehramtsstudium. Unterrichtswissenschaft, 47(1), 1-6. https://doi.org/10.1007/s420 10-018-00035-2

Kleickmann, T., Tröbst, S., Heinze, A., Bernholt, A., Rink, R. \& Kunter, M. (2017). Teacher Knowledge Experiment: Conditions of the Development of Pedagogical Content Knowledge. In D. Leutner, J. Fleischer, J. Grünkorn, \& E. Klieme (Hrsg.), Competence Assessment in Education (S.111-129). Springer International Publishing. https://doi.org/10.1007/978-3-319-50030-0_8

König, J., Drahmann, M. \& Rothland, M. (2018). Motivprofile von Studierenden zu Beginn der Lehrerbildung: Anwendung und Validierung eines personenzentrierten Ansatzes in Deutschland und Österreich. Zeitschrift für Bildungsforschung, 8(2), 153 -171. https://doi.org/10.1007/s35834-018-0212-0

König, J., Rothland, M., Darge, K., Lünnemann, M. \& Tachtsoglou, S. (2013). Erfassung und Struktur berufswahlrelevanter Faktoren für die Lehrerausbildung und den Lehrerberuf in Deutschland, Österreich und der Schweiz. Zeitschrift für Erziehungswissenschaft, 16(3), 553 -577. https://doi.org/10.1007/s11618-0130373-5

Krauss, S., Bruckmaier, G., Lindl, A., Hilbert, S., Binder, K., Steib, N. \& Blum, W. (2020). Competence as a continuum in the COACTIV study: The "cascade model". ZDM, 52(2), 311-327. https://doi. org/10.1007/s11858-020-01151-z

Künsting, J. \& Lipowsky, F. (2011). Studienwahlmotivation und Persönlichkeitseigenschaften als Prädiktoren für Zufriedenheit und Strategienutzung im Lehramtsstudium. Zeitschrift für Pädagogische Psychologie, 25(2), 105 -114. https://doi.org/10.1024 /1010-0652/a000038

Kunter, M. (2011). Motivation als Teil der professionellen Kompetenz-Forschungsbefunde zum Enthusiasmus von Lehrkräften. In Professionelle Kompetenz von Lehrkräften. Ergebnisse des Forschungsprogramms COACTIV. (Fachportal Pädagogik; S. 259 - 275). Waxmann. http://www.ciando.com/ebook/bid-229 459/intRefererID/241664

Kunter, M. \& Pohlmann, B. (2009). Lehrer. In E. Wild \& J. Möller (Hrsg.), Pädagogische Psychologie (S. 261 - 282). Springer Berlin Heidelberg. https://doi.org/10.1007/978-3-540-88573-3_11

Liebendörfer, M. (2018). Motivationsentwicklung im Mathematikstudium. Springer Spektrum.

Lin, E., Shi, Q., Wang, J., Zhang, S. \& Hui, L. (2012). Initial motivations for teaching: Comparison between preservice teachers in the United States and China. Asia-Pacific Journal of Teacher Education, 40(3), 227-248. https://doi.org/10.1080/135986 6X.2012.700047

Mair, P. (2018). Modern Psychometrics with R. Springer International Publishing. https://doi.org/10.1007/978-3-319-93177-7

McLean, L., Taylor, M. \& Jimenez, M. (2019). Career choice motivations in teacher training as predictors of burnout and career 
optimism in the first year of teaching. Teaching and Teacher Education, 85, 204-214. https://doi.org/10.1016/j.tate.2019. 06.020

Micknass, A., Ohlemann, S., Pfetsch, J. \& Ittel, A. (2019). Berufswahlmotive von Studierenden des beruflichen Lehramts. Bildung = Berufsbildung?!, 185-198. https://doi.org/10.3278/ $6004660 w 185$

Neugebauer, M. (2013). Wer entscheidet sich für ein Lehramtsstudium - und warum? Eine empirische Überprüfung der These von der Negativselektion in den Lehrerberuf. Zeitschrift für Erziehungswissenschaft, 16(1),157-184. https://doi.org/10.1007/ s11618-013-0343-y

Neugebauer, M. (2015). Who chooses teaching under different labor market conditions? Evidence from West Germany, 1980-2009. Teaching and Teacher Education, 45, 137-148. https://doi. org/10.1016/j.tate.2014.10.004

Pohlmann, B. \& Möller, J. (2010). Fragebogen zur Erfassung der Motivation für die Wahl des Lehramtsstudiums (FEMOLA). Zeitschrift für Pädagogische Psychologie, 24(1), 73-84. https:// doi.org/10.1024/1010-0652.a000005

Rach, S. (2019). Lehramtsstudierende im Fach Mathematik -Wie hilft uns die Analyse von Lernvoraussetzungen für eine kohärente Lehrerbildung? In K. Hellmann, J. Kreutz, M. Schwichow, \& K. Zaki (Hrsg.), Kohärenz in der Lehrerbildung (S.69-84). Springer Fachmedien Wiesbaden. https://doi.org/10.1007/9783-658-23940-4_5

Reichl, C., Wach, F., Spinath, F. M., Brünken, R. \& Karbach, J. (2014). Burnout risk among first-year teacher students: The roles of personality and motivation. Journal of Vocational Behavior, 85(1), $85-92$.

Retelsdorf, J., Bauer, J., Gebauer, S. K., Kauper, T. \& Möller, J. (2014). Erfassung berufsbezogener Selbstkonzepte von angehenden Lehrkräften (ERBSE-L). Diagnostica, 60(2), 98 -110. https://doi. org/10.1026/0012-1924/a000108

Retelsdorf, J. \& Möller, J. (2012). Grundschule oder Gymnasium? Zur Motivation ein Lehramt zu studieren. Zeitschrift für Pädagogische Psychologie, 26(1), 005 - 017. https://doi.org/10.1024/ 1010-0652/a000056

Rheinberg, F. \& Vollmeyer, R. (2018). Motivation. Kohlhammer Verlag.

Richardson, P. W. \& Watt, H. M. G. (2006). Who Chooses Teaching and Why? Profiling Characteristics and Motivations Across Three Australian Universities. Asia-Pacific Journal of Teacher Education, 34(1), 27-56. https://doi.org/10.1080/1359866 0500480290

Roloff Henoch, J., Klusmann, U., Lüdtke, O. \& Trautwein, U. (2015). Who becomes a teacher? Challenging the "negative selection" hypothesis. Learning and Instruction, 36, 46-56. https://doi. org/10.1016/j.learninstruc.2014.11.005

Rosseel, Y. (2019). Lavaan: Latent Variable Analysis. https://cran.rproject.org/web/packages/lavaan/

Rothland, M. (2013). „Riskante“ Berufswahlmotive und Überzeugungen von Lehramtsstudierenden. Erziehung und Unterricht, $71-80$.

Rutsch, J., Spinath, B., Rehm, M., Vogel, M. \& Dörfler, T. (2020). Unterscheiden sich Sonderpädagogikstudierende bezüglich Berufswahlmotivation und pädagogischer Vorerfahrungen von Primarstufen- und Sekundarstufenstudierenden? Zeitschrift für Pädagogische Psychologie, 34(2), 87-98. https://doi. org/10.1024/1010-0652/a000258

Ryan, R. M. \& Deci, E. L. (2000). Intrinsic and Extrinsic Motivations: Classic Definitions and New Directions. Contemporary Educational Psychology, 25(1), 54-67. https://doi.org/10.1006/ceps. 1999.1020
Schendera, C. F. G. (2010). Clusteranalyse mit SPSS: Mit Faktorenanalyse. Oldenbourg.

Sinclair, C. (2008). Initial and changing student teacher motivation and commitment to teaching. Asia-Pacific Journal of Teacher Education, 36(2), 79-104. https://doi.org/10.1080/13598660 801971658

Spinath, B., van Ophuysen, S. \& Heise, E. (2005). Individuelle Voraussetzungen von Studierenden zu Studienbeginn: Sind Lehramtsstudierende so schlecht wie ihr Ruf? Psychologie in Erziehung und Unterricht, 3, 186-197.

Thomson, M. M., Turner, J. E. \& Nietfeld, J. L. (2012). A typological approach to investigate the teaching career decision: Motivations and beliefs about teaching of prospective teacher candidates. Teaching and Teacher Education, 28(3), 324-335. https:// doi.org/10.1016/j.tate.2011.10.007

Ufer, S., Rach, S. \& Kosiol, T. (2017). Interest in mathematics = interest in mathematics? What general measures of interest reflect when the object of interest changes. ZDM, 49(3), 397- 409. https://doi.org/10.1007/s11858-016-0828-2

Wach, F.-S., Karbach, J., Ruffing, S., Brünken, R. \& Spinath, F. M. (2016). University Students' Satisfaction with their Academic Studies: Personality and Motivation Matter. Frontiers in Psychology, 7. https://doi.org/10.3389/fpsyg.2016.00055

Watt, H. M. G., Richardson, P. W., Klusmann, U., Kunter, M., Beyer, B., Trautwein, U. \& Baumert, J. (2012). Motivations for choosing teaching as a career: An international comparison using the FIT-Choice scale. Teaching and Teacher Education, 28(6), 791 - 805. https://doi.org/10.1016/j.tate.2012.03.003

Watt, H. M. G., Richardson, P. W. \& Morris, Z. A. (2017). Divided by discipline? Contrasting motivations, perceptions, and background characteristics of beginning Australian English and mathematics teachers. In Global perspectives on teacher motivation (S. 349 - 376). Cambridge University Press.

Wigfield, A., Eccles, J. S., Fredricks, J. A., Simpkins, S., Roeser, R. W. \& Schiefele, U. (2015). Development of Achievement Motivation and Engagement. In R. M. Lerner (Hrsg.), Handbook of Child Psychology and Developmental Science (S.1-44). John Wiley \& Sons, Inc. https://doi.org/10.1002/9781118963418.childpsy316

Windolf, P. (1992). Fachkultur und Studienfachwahl Ergebnisse einer Befragung von Studienanfängern. Kölner Zeitschrift für Soziologie und Sozialpsychologie, 44(1), $76-98$.

\section{Historie}

Manuskript eingereicht: 15.09.2020

Manuskript nach Revision angenommen: 19.03.2021

Onlineveröffentlichung: 28.04.2021

\section{Förderung}

Open-Access-Veröffentlichung ermöglicht durch die Leuphana Universität.

\section{ORCID}

Robin Göller

(D) https://orcid.org/0000-0001-9626-0314

\section{Robin Göller}

Leuphana Universität Lüneburg

Institut für Mathematik und ihre Didaktik

Universitätsallee 1

21335 Lüneburg

Deutschland

robin.goeller@leuphana.de 